\title{
Pacific
}

Journal of

Mathematics

\section{ONE-PARAMETER FIXED POINT INDICES}

DONCO DIMOVSKI

Volume 164 No. 2

June 1994 


\section{ONE-PARAMETER FIXED POINT INDICES}

\section{DoNČo DimovsKi}

Let $F: X \times I \rightarrow X$ be a PL homotopy, where $X$ is a compact connected PL $n$-dimensional manifold, in the euclidean space $\mathbb{R}^{n}$, $n \geq 4$, and let $P: X \times I \rightarrow X$ be the projection. A fixed point of $F$ is a point $(x, t) \in X \times I$ such that $F(x, t)=x$. The set of all the fixed points of $F$ is denoted by $\operatorname{Fix}(F)$. For a family $V$ of isolated circles of fixed points of $F$ we define two indices: $\operatorname{ind}_{1}(F, V)$ which is an element in the first homology group $H_{1}(E)$, where $E$ is the space of paths in $X \times I \times X$ from the graph of $F$ to the graph of $P$; and $\operatorname{ind}_{2}(F, V)$-which is an element in the group $\mathbb{Z}_{2}$ with two elements. We prove that there is a compact neighborhood $N$ of $V$ and a homotopy from $F$ to $H$ rel $X \times I \backslash N$ such that $\operatorname{Fix}(H)=$ $\operatorname{Fix}(F) \backslash V$ if and only if $\operatorname{ind}_{1}(V, F)=0$ and $\operatorname{ind}_{2}(V, F)=0$. The indices $\operatorname{ind}_{1}(V, f)$ and $\operatorname{ind}_{2}(V, F)$ are defined via the degrees, $\operatorname{deg}_{1}(g)$ and $\operatorname{deg}_{2}(g)$, for maps $g: S^{1} \times S^{m} \rightarrow S^{m}$. Moreover, we show how to modify $F$ to create circles of fixed points with prescribed indices.

Introduction. In this paper we define two indices for fixed points of homotopies between two selfmaps of a manifold, and then show that these indices provide us with sufficient and necessary conditions for removing some or all of the fixed point set, in a controlled manner. Let $F: X \times I \rightarrow X$ be a PL homotopy, where $X$ is a compact connected PL $n$-dimensional manifold, contained in the euclidean space $\mathbb{R}^{n}$, let $n \geq 4$, and let $P: X \times I \rightarrow X$ be the projection. A fixed point of $F$ is a point $(x, t) \in X \times I$ such that $F(x, t)=x$. The set of all the fixed points of $F$ is denoted by $\operatorname{Fix}(F)$. In this setting, isolated circles of fixed points are the generic form of fixed points, as isolated individual fixed points are in the classical setting. The two indices, $\operatorname{ind}_{1}(F, V)$ and $\operatorname{ind}_{2}(F, V)$, are defined for a family $V$ of finitely many isolated circles of fixed points of $F$. The first index, ind $_{1}(F, V)$, is an element in the first homology group $H_{1}(E)$, where $E$ is the space of paths in $X \times I \times X$ from the graph of $F$ to the graph of $P$, and is a slight generalization of the first obstruction discussed in [DG]. It is mentioned in [DG] that a solution to the one parameter fixed point problem in the transverse case can be found in [HQ], via an obstruction lying in the 1-dimensional framed bordism group of 
the function space $E$. This obstruction for a family of finitely many isolated transverse circles of fixed points $V$ is zero if and only if ind $_{1}(F, V)=0$ and $\operatorname{ind}_{2}(F, V)=0$. The second index, $\operatorname{ind}_{2}(F, V)$, is an element of the group with two elements $\mathbb{Z}_{2}$, and corresponds to the second obstruction given in [DG], but the second obstruction in [DG] can be defined only if we know that the first obstruction is zero, while $\operatorname{ind}_{2}(F, V)$ is defined independently of $\operatorname{ind}_{1}(F, V)$. The main improvement of the results from [DG] is the following Theorem 4.3.

THEOREM 4.3. There is a compact neighborhood $N$ of $V$ and a homotopy from $F$ to $H \operatorname{rel} X \times I \backslash N$ such that $\operatorname{Fix}(H)=\operatorname{Fix}(F) \backslash V$ if and only if $\operatorname{ind}_{1}(V, F)=0$ and $\operatorname{ind}_{2}(V, F)=0$.

Other results in this paper are: joining circles of fixed points; homotoping $F$ to $H$, such that each fixed point class of $H$ is a transverse circle of fixed points; and creating circles of fixed points, with prescribed indices.

The indices $\operatorname{ind}_{1}(V, F)$ and $\operatorname{ind}_{2}(V, F)$ are defined via the degrees $\operatorname{deg}_{1}(g)$ and $\operatorname{deg}_{2}(g)$ for maps $g: S^{1} \times S^{m} \rightarrow S^{m}$.

The paper is organized as follows.

In part I the notions of degrees, $\operatorname{deg}_{1}(g)$ and $\operatorname{deg}_{2}(g)$ for maps $g: S^{1} \times S^{m} \times S^{m}$, are introduced, where $\operatorname{deg}_{1}(g)$ is an element from the group of integers $\mathbb{Z}$, and $\operatorname{deg}_{2}(g)$ is an element from $\mathbb{Z}_{2}$. One of the results about these degrees is the fact that two maps $g, g^{\prime}: S^{1} \times S^{m} \rightarrow$ $S^{m}$ are homotopic if and only if $\operatorname{deg}_{1}(g)=\operatorname{deg}_{1}\left(g^{\prime}\right)$ and $\operatorname{deg}_{2}(g)=$ $\operatorname{deg}_{2}\left(g^{\prime}\right)$.

In part II three standard models, i.e. standard regular neighborhoods of a disk, an annulus and a disk with two holes in $\mathbb{R}^{m+1}$ are defined, and several facts about the extensions of maps from subsets of these standard models into $S^{m}$ to the entire model are proven.

In part III the notions of $i_{1}(F, C)$ and $i_{2}(F, C)$ are introduced, where $C$ is an isolated circle of fixed points, as $\mathrm{deg}_{1}$ and $\mathrm{deg}_{2}$ of the restriction of $P-F$ to the boundary of the neighborhood of the circle $C$, where $P: X \times I \rightarrow X$ is the projection, and $P-F$ is considered as a map into $\mathbb{R}^{n}$. Later, it is shown how to remove and create circles of fixed points, using the standard models from part II.

Part IV contains the main results. First, Theorem 4.3 is proven, and then it is shown how to homotope the map to a new map, each fixed point class of which consists of a single circle.

We are concerned in this paper only with the fixed points of homotopies between selfmaps of $X$, where $X$ is an $n$-manifold in $\mathbb{R}^{n}$. 
The general case of coincidences between two maps $F, G: M \rightarrow N$, where $M$ is an $(m+1)$-dimensional and $N$ is an $m$-dimensional manifold, using the same techniques as in this paper will be discussed in a subsequent paper. For this we need a geometric description of spin manifolds, which is going to appear in a joint paper with Ross Geoghegan. The general case has also been considered in a paper by Jerzy Jezierski $[\mathbf{J}]$.

At the end of this introduction, I would like to make known my gratitude to Helga Schirmer and to thank her not only for the valuable discussions that we had, but also for her hospitality and financial support during my visit to University of Carleton, where the work in this paper began and most of it was done. Also, I would like to thank Ross Geoghegan for the numerous helpful conversations on this subject.

I. Degrees 1 and 2. For a positive integer $m$ we denote by $\mathbf{x}$ the element $\left(x_{1}, x_{2}, \ldots, x_{m}\right)$ of $\mathbb{R}^{m}$ and by $|\mathbf{x}|$ the length $\sqrt{x_{1}^{2}+x_{2}^{2}+\cdots+x_{m}^{2}}$ of $\mathbf{x}$. If $\mathbf{x}=\left(x_{1}, x_{2}, \ldots, x_{m}\right) \in \mathbb{R}^{m}$ and $\mathbf{y}=$ $\left(y_{1}, \ldots, y_{n}\right) \in \mathbb{R}^{n}$, then $(\mathbf{x}, \mathbf{y})$ will denote the element $\left(x_{1}, \ldots, x_{m}\right.$, $\left.y_{1}, \ldots, y_{n}\right) \in \mathbb{R}^{m+n}$. If $\mathbf{x}=\left(x_{1}, x_{2}, \ldots, x_{m}\right) \in \mathbb{R}^{n}$ and $r \in \mathbb{R}$, then $r \mathbf{x}=\left(r x_{1}, \ldots, r x_{m}\right)$. Let $D^{m}=\left\{\mathbf{x}\left|\mathbf{x} \in \mathbb{R}^{m},\right| \mathbf{x} \mid \leq 1\right\}$ and $S^{m}=\left\{\mathbf{x}\left|\mathbf{x} \in \mathbb{R}^{m+1},\right| \mathbf{x} \mid=1\right\}$. We choose once and for all an orientation of $\mathbb{R}^{m}$, for all $m$, called the standard orientation, such that the standard orientation on $\mathbb{R}^{m+1}=\mathbb{R}^{m} \times \mathbb{R}$ is the product of the standard orientations on $\mathbb{R}^{m}$ and $\mathbb{R}$. We assume that $D^{m}$, and $I=[0,1]$ are oriented by the induced orientations from $\mathbb{R}^{m}$ and $\mathbb{R}$. Let $S^{m}$ have the induced orientation from $\mathbb{R}^{m+1}$ and let $S^{1} \times S^{m}$ and $S^{1} \times D^{m}$ have the product orientations.

Definition 1.1. Let $f: S^{1} \times S^{m} \rightarrow S^{m}$ be a given map. We define $\operatorname{deg}_{1}(f)=\operatorname{deg}(\varphi)$, where $\varphi: S^{m} \rightarrow S^{m}$ is defined by $\varphi(\mathbf{x})=f(P, \mathbf{x})$ for a point $P \in S^{1}$, and deg is the usual degree of a map from $S^{m}$ to $S^{m}$.

Fact 1.1. $\operatorname{deg}_{1}(f)$ is well defined.

Proof. Let $\varphi(\mathbf{x})=f(P, \mathbf{x})$ and $\psi(\mathbf{x})=f(Q, \mathbf{x})$ for two points $P, Q \in S^{1}$. Let $g:[0,1] \rightarrow[P, Q]$ be a homeomorphism from the interval $I=[0,1]$ to one of the arcs from $P$ to $Q$ in $S^{1}$. Then $H(\mathbf{x}, t)=f(g(t), \mathbf{x})$ defines a homotopy from $\varphi$ to $\psi$. Hence $\operatorname{deg}(\varphi)=\operatorname{deg}(\psi)$. 


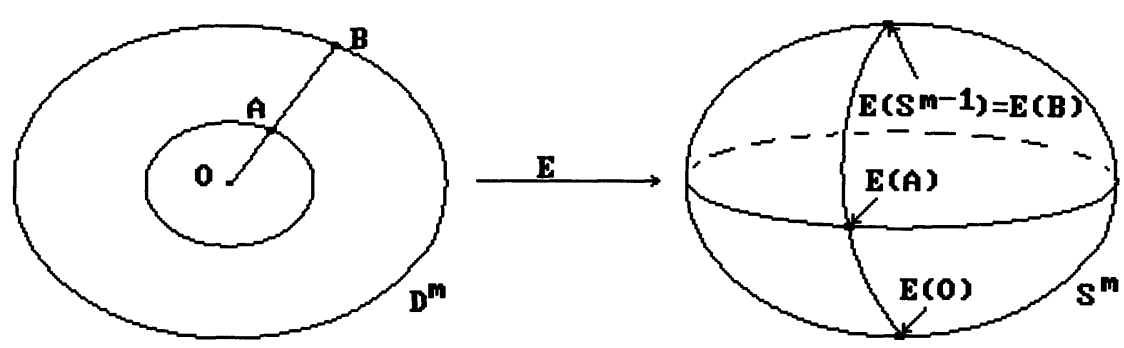

FIGURE 1.1

For a given $m$, we define a map $E: D^{m} \rightarrow S^{m}$, by:

$$
E(\mathbf{x})=\left(\alpha \mathbf{x}, \varepsilon \sqrt{1-(\alpha|\mathbf{x}|)^{2}}\right)
$$

where: $\alpha=2, \varepsilon=-1$ for $0 \leq|\mathbf{x}| \leq \frac{1}{2}$, and $\alpha=2 \cdot \frac{1-|\mathbf{x}|}{|\mathbf{x}|}, \varepsilon=1$ for $\frac{1}{2} \leq|\mathbf{x}| \leq 1$. The map is well defined because $|\alpha \mathbf{x}|^{2}+\left(1-(\alpha|\mathbf{x}|)^{2}\right)=1$. A schematic picture of the map $E$ is given in Figure 1.1 , for $m=2$.

Let $\mathcal{N}(m)$ be the point $(0,0, \ldots, 0,1) \in S^{m}$, i.e. the "north pole". It is easy to check that $E\left(\partial D^{m}\right)=\mathscr{N}(m)$ and the restriction $E \mid$ is a homeomorphism from int $D^{m}$ to $S^{m} \backslash\{\mathscr{N}(m)\}$.

Let $X$ be the factor space obtained from $S^{1} \times S^{m} \cup D^{2}$ by the identification of $(\mathbf{x}, \mathscr{N}(m)) \in S^{1} \times S^{m}$ with $\mathbf{x} \in S^{1}=\partial D^{2}$. For each $t \in(0,1]$ we will define a map $K_{t}: S^{m+1} \rightarrow x$ as follows. Consider $S^{m+1}$ as a subset of $D^{2} \times D^{m}$ and for $(\mathbf{z}, \mathbf{y}) \in S^{m+1}$, with $\mathbf{z} \in D^{2}$ and $\mathbf{y} \in D^{m}$, we define

(1.2) $K_{t}(\mathbf{z}, \mathbf{y})= \begin{cases}\frac{2}{t} \mathbf{z} \in D^{2} & \text { if } 0 \leq|\mathbf{z}| \leq \frac{t}{2} \\ \left(\frac{\mathbf{z}}{|\mathbf{z}|}, E\left(\frac{2}{\sqrt{4-t^{2}}} \cdot \mathbf{y}\right)\right) \in S^{1} \times S^{m} & \text { if } \frac{t}{2} \leq|\mathbf{z}| \leq 1\end{cases}$

These maps are well defined because for $t \in(0,1]$ and $|\mathbf{z}|=\frac{t}{2}$, we have $\frac{2}{t} \mathbf{z}=\frac{\mathbf{z}}{|\mathbf{z}|} \in \partial D^{2}$ and $|\mathbf{y}|=1-\left(\frac{t}{2}\right)^{2}$, i.e. $\frac{2}{t} \mathbf{z}=\left(\frac{2}{t} \mathbf{z}, \mathscr{N}(m)\right)=$ $\left(\frac{\mathbf{z}}{|\mathbf{z}|}, E\left(\frac{2}{\sqrt{4-t^{2}}} \cdot \mathbf{y}\right)\right)$. We denote $K_{1}$ by $K$. A schematic picture for the space $X$ and the map $K$, when $m=1$, is given in Figure 1.2.

Definition 1.2. Let $f: S^{1} \times S^{m} \rightarrow S^{m}$ be a given map. We define $\operatorname{deg}_{2}(f)$ to be the element $[\bar{f} \circ K]$ from $\pi_{m+1}\left(S^{m}\right)=\mathbb{Z}_{2}$ where $\bar{f}: X \rightarrow S^{m}$ is defined by $f$ on $S^{1} \times S^{m}$ and by an extension on $D^{2}$ of $f$ restricted to $\partial D^{2}$.

Fact 1.2. For $m \geq 3, \operatorname{deg}_{2}(f)$ is well defined. 


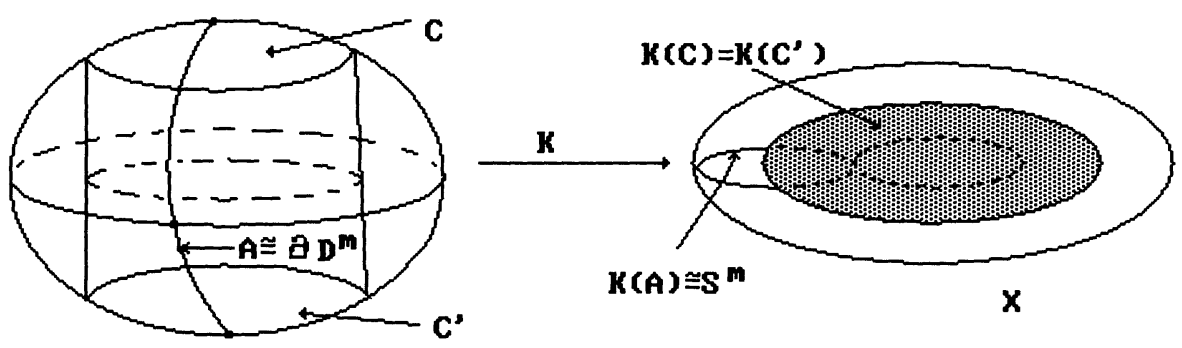

FIGURE 1.2

Proof. The only choice in the definition of $\operatorname{deg}_{2}(f)$ is the choice of the extension on $D^{2}$ of the restriction of $f$ to $\partial D^{2}$. Let $f_{i}: X \rightarrow S^{m}$ be defined by $f$ on $S^{1} \times S^{m+1}$ and by $\alpha_{i}$ on $D^{2}$, for two such different extensions on $D^{2}, \alpha_{1}$ and $\alpha_{2}$. The maps $\alpha_{1}$ and $\alpha_{2}$ define a map $g: S^{2} \rightarrow S^{m}$. For $m \geq 3$, since $\pi_{2}\left(S^{m}\right)=0$, this map has an extension $h: D^{3} \rightarrow S^{m}$. This extension $h$ gives a homotopy from $f_{1} \circ K$ to $f_{2} \circ K$. So, $\operatorname{deg}_{2}(f)$ is well defined.

Proposition 1.3. Let $f, g: S^{1} \times S^{m} \rightarrow S^{m}$ be homotopic maps. Then, for $m \geq 3, \operatorname{deg}_{1}(f)=\operatorname{deg}_{1}(g)$, and $\operatorname{deg}_{2}(f)=\operatorname{deg}_{2}(g)$.

Proof. Suppose that $H: S^{1} \times S^{m} \times I \rightarrow S^{m}$ is the given homotopy. Then, the restriction of $H$ to $\{P\} \times S^{m} \times I$ gives a homotopy for the restrictions of $f$ and $g$ to $\{P\} \times S^{m}$, which shows that $\operatorname{deg}_{1}(f)=$ $\operatorname{deg}_{1}(g)$.

Next, let $\mathscr{N}(m), X, K, \bar{f}$, and $\bar{g}$ be as defined above. Now, we can extend the homotopy $H$ to a homotopy $\bar{H}: X \times I \rightarrow S^{m}$, by an extension of the restriction of $H$ to $\left(S^{1} \times\{\mathscr{N}(m)\} \times I\right) \cup\left(D^{2} \times\right.$ $\{0\}) \cup\left(D^{2} \times\{1\}\right)$. Such an extension exists, because $\left(S^{1} \times\{\mathscr{N}(m)\} \times\right.$ I) $\cup\left(D^{2} \times\{0\}\right) \cup\left(D^{2} \times\{1\}\right)$ is in fact an $S^{2}$, and we have a map from $S^{2}$ to $S^{m}$, and for $m \geq 3$, all such maps have an extension. In this way we have obtained a homotopy $\bar{H}$ from $\bar{f}$ to $\bar{g}$. Then the composition $\bar{H} \circ(K \times$ id $): S^{m+1} \times I \rightarrow X \times I \rightarrow S^{m}$ gives a homotopy for the compositions $\bar{f} \circ K$ and $\bar{g} \circ K$. Now, since $[\bar{f} \circ K]=0$ is equivalent to the fact that $\bar{f} \circ K$ has an extension over $D^{m+2}$ which is equivalent by the above homotopy $\bar{H} \circ(K \times I)$ to the fact that $\bar{g} \circ K$ has an extension over $D^{m+2}$, which is equivalent to the fact that $[\bar{g} \circ K]=0$, we have that $[\bar{f} \circ K]=[\bar{g} \circ K]$ in $\pi_{m+1}\left(S^{m}\right)$. Hence, $\operatorname{deg}_{2}(f)=\operatorname{deg}_{2}(g)$.

REMARK 1.4. For $f: S^{1} \times S^{m} \rightarrow S^{m}$ and $m \geq 3$, since the restriction of $f$ to $S^{1} \times\{\mathscr{N}(m)\}$ is homotopic to a constant map, it 
follows that $f$ is homotopic to a map $g: S^{1} \times S^{m} \times S^{m}$, such that $g\left(S^{1} \times\{\mathscr{N}(m)\}\right)=Q$, for some $Q \in S^{m}$. Then in the definition of $\operatorname{deg}_{2}(g)$ we can choose the constant homotopy on $D^{2}$, i.e. we can define $\bar{g}\left(D^{2}\right)=Q$. This says that $\bar{g} \circ K(\mathbf{z}, \mathbf{y})=Q$ for every $(\mathbf{z}, \mathbf{y}) \in S^{m+1}$ with $\mathbf{z} \in D^{2}, \mathbf{y} \in D^{m}$, and $|\mathbf{z}| \leq \frac{1}{2}$.

Next, let $f: S^{1} \times S^{m} \rightarrow S^{m}$, be such that $f\left(S^{1} \times\{\mathscr{N}(m)\}\right)=Q$, for some point $Q \in S^{m}$. We define a map $F(f): S^{m+1} \rightarrow S^{m}$, as follows. For $(\mathbf{z}, \mathbf{y}) \in S^{m+1}$ with $\mathbf{z} \in D^{2}, \mathbf{y} \in D^{m}$, let:

$$
F(f)(\mathbf{z}, \mathbf{y})= \begin{cases}f\left(\frac{\mathbf{z}}{|\mathbf{z}|}, E(\mathbf{y})\right) & \text { for } \mathbf{z} \neq \mathbf{0}, \\ Q & \text { for } \mathbf{z}=\mathbf{0} .\end{cases}
$$

This map is well defined, because $f\left(S^{1} \times\{\mathscr{N}(m)\}\right)=Q$, and $E\left(\partial D^{m}\right)$ $=\mathscr{N}(m)$. The map $F(f)$ may be described as a union of two maps: $F_{1}: S^{m+1} \backslash\left(\{0\} \times D^{m}\right) \rightarrow S^{m}$ and $F_{2}:\{0\} \times D^{m} \rightarrow S^{m}$, where $S^{m+1}$ is considered as a subset of $D^{2} \times D^{m}$ and 0 is in $D^{2}$. The map $F_{1}$ is the composition $f \circ(1 \times E) \circ h$, where $h: S^{m+1} \backslash\left(\{0\} \times D^{m}\right) \rightarrow S^{1} \times D^{m}$ is defined by: $h(\mathbf{z}, \mathbf{y})=\left(\frac{\mathbf{z}}{|\mathbf{z}|}, \mathbf{y}\right)$, and $F_{2}\left(\{\mathbf{0}\} \times D^{m}\right)=Q$ :

Proposition 1.5. Let $f: S^{1} \times S^{m} \rightarrow S^{m}$, and $F(f): S^{m+1} \rightarrow S^{m}$ be as above. Then, for $m \geq 3, \operatorname{deg}_{2}(f)=[F(f)] \in \pi_{m+1}\left(S^{m}\right)$.

Proof. By the above Remark 1.4, in the definition of $\operatorname{deg}_{2}(f)$ we can choose the map $\bar{f}$ such that $\bar{f}\left(D^{2}\right)=Q$. It can be checked that $\lim _{t \rightarrow 0} \bar{f} \circ K_{t}(\mathbf{z}, \mathbf{y})=F(f)(\mathbf{z}, \mathbf{y})$. So,

$$
H(\mathbf{z}, \mathbf{y}, t)= \begin{cases}\bar{f} \circ K_{t}(\mathbf{z}, \mathbf{y}) & \text { for } 0<t \leq 1, \\ F(f)(\mathbf{z}, \mathbf{y}) & \text { for } t=0\end{cases}
$$

is well defined homotopy from $F(f)$ to $\bar{f} \circ K$. Hence, $\operatorname{deg}_{2} f=$ $[F(f)]$.

Let $p: S^{1} \times S^{m} \rightarrow S^{m}$ be the projection, $p(\mathbf{z}, \mathbf{y})=\mathbf{y}$.

Proposition 1.6. Let $\varphi: S^{m} \rightarrow S^{m}$ be a given map, and let $g: S^{1} \times$ $S^{m} \rightarrow S^{m}$ be defined by $g=\varphi \circ p$. Then, $\operatorname{deg}_{2}(g)=0$.

Proof. The map $E F(g): D^{m+2} \rightarrow S^{m}$ defined by $E F(g)(\mathbf{z}, \mathbf{y})=$ $\varphi(E(\mathbf{y}))$ for $\mathbf{z} \in D^{2}, \mathbf{y} \in D^{m}$ is well defined and is an extension of the map $F(g)$, which shows that $[F(g)]=0$ in $\pi_{m+1}\left(S^{m}\right)$, i.e. $\operatorname{deg}_{2}(g)=0$.

Proposition 1.7. Let $f: S^{1} \times S^{m} \rightarrow S^{m}$ be such that $f\left(S^{1} \times\{\mathscr{N}(m)\}\right)$ $=Q$ for some $Q \in S^{m}$, and let $m \geq 3$. Let $g: S^{1} \times S^{m} \rightarrow S^{m}$ 
be the map defined by $g(\mathbf{z}, \mathbf{y})=f(P, \mathbf{y})$ for $\mathbf{z} \in S^{1}, \mathbf{y} \in S^{m}$, where $P$ is a point of $S^{1}$. Then, $\operatorname{deg}_{2}(f)=0$ if and only if $f$ is homotopic to the map $g$.

Proof. Let $f$ be homotopic to $g$. Then $\operatorname{deg}_{2}(f)=\operatorname{deg}_{2}(g)$ by Proposition 1.3, and $\operatorname{deg}_{2}(f)=0$ by Proposition 1.6.

Conversely, let $\operatorname{deg}_{2}(f)=0$. Then the map $F(f)$ has an extension $E(f): D^{m+2} \rightarrow S^{m}$. We define a map $g: S^{1} \times S^{m} \rightarrow S^{m}$ as follows. For $(\mathbf{z}, \mathbf{y}) \in S^{1} \times S^{m}$, we define $g(\mathbf{z}, \mathbf{y})=E(f)\left(\mathbf{0}, E^{-1}(\mathbf{y})\right)$, for $\mathbf{y} \neq \mathscr{N}(m)$ and $g(\mathbf{z}, \mathscr{N}(m))=Q$, where $E: D^{m} \rightarrow S^{m}$ is the map defined by (1.1). This map is well defined, because $E$ is a homeomorphism from int $D^{m}$ to $S^{m} \backslash\{\mathscr{N}(m)\}$, maps $\partial D^{m}$ onto $\mathscr{N}(m)$, and $f\left(S^{1} \times\{\mathcal{N}(m)\}\right)=Q$. Moreover, $g(\mathbf{z}, \mathbf{y})=f(P, \mathbf{y})$ for $\mathbf{z} \in S^{1}$ and $\mathbf{y} \in S^{m}$, where $P$ is a point of $S^{1}$. Proposition 1.6 implies that $\operatorname{deg}_{2}(g)=0$.

Next, we will show that $f$ is homotopic to $g$. First we define a map $G: S^{1} \times D^{m} \times I \rightarrow D^{m+2}$ as follows. For $\mathbf{z} \in S^{1}, \mathbf{y} \in D^{m}, r \in I$,

$$
G(\mathbf{z}, \mathbf{y}, r)=(\lambda \mathbf{z}, \mathbf{y})
$$

where

$$
\lambda=\frac{r^{2}-1+\sqrt{\left(r^{2}-1\right)^{2}-4 r^{2}|\mathbf{y}|^{2}+4 r^{2}}}{2 r},
$$

for $r \neq 0$, and $\lambda=0$ for $r=0$. This map is well defined because the limit of $\lambda$ when $r$ goes to 0 is 0 . The map $G$ is shown schematically in Figure 1.3 (next page). For $\mathbf{y} \in S^{m-1}=\partial D^{m}$, i.e. when $|\mathbf{y}|=1$, we have

$$
\lambda=\frac{r^{2}-1+\sqrt{\left(r^{2}-1\right)^{2}}}{2 r}=0
$$

and so, $G(\mathbf{z}, \mathbf{y}, r)=(\mathbf{0}, \mathbf{y})$ for $|\mathbf{y}|=1$.

Now we define a map $H: S^{1} \times S^{m} \times I \rightarrow S^{m}$ by:

$$
H(\mathbf{z}, \mathbf{y}, r)=E(f)\left(G\left(\mathbf{z}, E^{-1}(\mathbf{y}), r\right)\right) .
$$

This map is well defined, because $E(f)(G(\mathbf{z}, \mathbf{u}, r))=E(f)(\mathbf{0}, \mathbf{u})=$ $F(f)(\mathbf{0}, \mathbf{u})$, for each $\mathbf{u} \in E^{-1}(\mathcal{N}(m))$, i.e. for each $\mathbf{u} \in D^{m}$ with $|\mathbf{u}|=1$. The map $H$ is continuous because $E$ is homeomorphism on int $D^{m}$, and when $\mathbf{y} \in S^{m}$ is close to $\mathscr{N}(m)$ then: $E^{-1}(\mathbf{y})$ is close to $\partial D^{m}$, i.e. $\left|E^{-1}(\mathbf{y})\right|$ is close to $1 ; \lambda$ is close to $0 ;|\lambda \mathbf{z}|$ is close to $0 ; G\left(\mathbf{z}, E^{-1}(\mathbf{y}), r\right)=\left(\lambda \mathbf{z}, E^{-1}(\mathbf{y})\right)$ is close to $\partial D^{m+2}=S^{m+1}$; $E(f)\left(\lambda \mathbf{z}, E^{-1}(\mathbf{y})\right)$ is close to $F(f)\left(\lambda \mathbf{z}, E^{-1}(\mathbf{y})\right) ;$ and $F(f)\left(\lambda \mathbf{z}, E^{-1}(\mathbf{y})\right)$ is close to $Q$. 


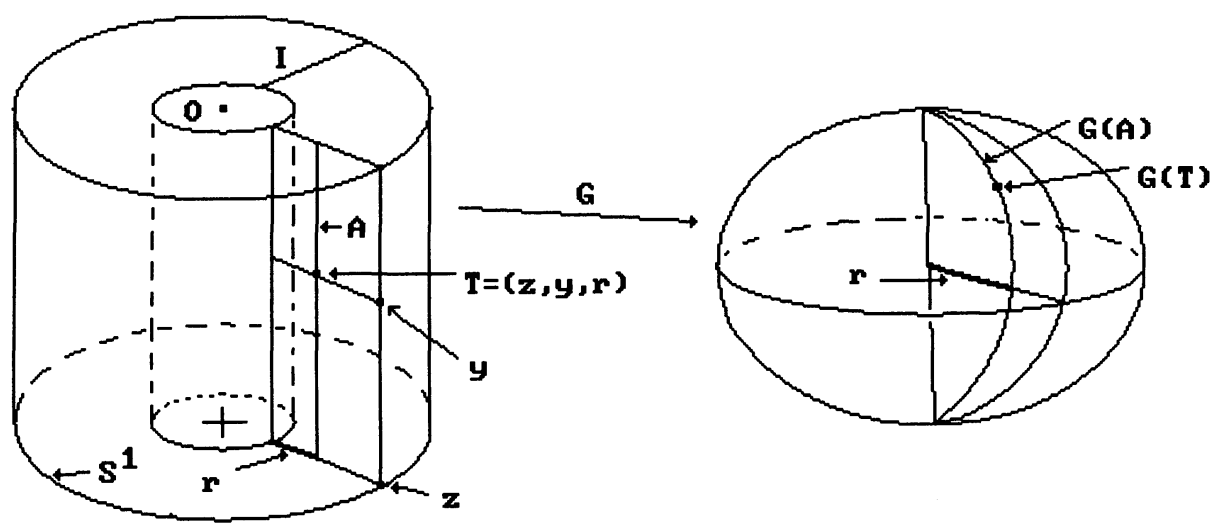

FIGURE 1.3

Next,

$$
H(\mathbf{z}, \mathbf{y}, 0)=E(f)\left(G\left(\mathbf{z}, E^{-1}(\mathbf{y}), 0\right)\right)=E(f)\left(\mathbf{0}, E^{-1}(\mathbf{y})\right)=g(\mathbf{z}, \mathbf{y}),
$$

and

$$
\begin{aligned}
H(\mathbf{z}, \mathbf{y}, 1) & =E(f)\left(G\left(\mathbf{z}, E^{-1}(y), 1\right)\right) \\
& =E(f)\left(\sqrt{\left(1-\left|E^{-1}(\mathbf{y})\right|^{2}\right.} \cdot \mathbf{z}, E^{-1}(\mathbf{y})\right) \\
& \left.=F(f)\left(\sqrt{\left(1-\left|E^{-1}(\mathbf{y})\right|^{2}\right.}\right) \cdot \mathbf{z}, E^{-1}(\mathbf{y})\right) \\
& =f\left(\mathbf{z}, E\left(E^{-1}(\mathbf{y})\right)\right)=f(\mathbf{z}, \mathbf{y}) .
\end{aligned}
$$

Hence, $f$ is homotopic to $g$.

Proposition 1.8. Let $m \geq 3$ and, for $f, g: S^{1} \times S^{m} \rightarrow S^{m}$, let $\operatorname{deg}_{1}(f)=\operatorname{deg}_{1}(g)$ and $\operatorname{deg}_{2}(f)=\operatorname{deg}_{2}(g)=0$. Then $f$ is homotopic to $g$.

Proof. By Proposition 1.7, $f$ and $g$ are homotopic to maps $f^{\prime}$ and $g^{\prime}$, such that $f^{\prime}(\mathbf{z}, \mathbf{x})=f^{\prime}(P, \mathbf{x})=f^{\prime \prime}(\mathbf{x}), g^{\prime}(\mathbf{z}, \mathbf{x})=g^{\prime}(P, \mathbf{x})=$ $g^{\prime \prime}(\mathbf{x}), \operatorname{deg}_{1}\left(f^{\prime}\right)=\operatorname{deg}_{1}(f)=\operatorname{deg}\left(f^{\prime \prime}\right)$, and $\operatorname{deg}_{1}\left(g^{\prime}\right)=\operatorname{deg}_{1}(g)=$ $\operatorname{deg}\left(g^{\prime \prime}\right)$ for every $\mathbf{z} \in S^{1}$ and $\mathbf{x} \in S^{m}$, where $f^{\prime \prime}, g^{\prime \prime}: S^{m} \rightarrow S^{m}$. Now, by Hopf's theorem, $f^{\prime \prime}$ is homotopic to $g^{\prime \prime}$. If $H: S^{m} \times I \rightarrow S^{m}$ is a homotopy between $f^{\prime \prime}$ and $g^{\prime \prime}$, then $H^{\prime}: S^{1} \times S^{m} \times I \rightarrow S^{m}$ defined by $H^{\prime}(\mathbf{z}, \mathbf{x}, t)=H(\mathbf{x}, t)$, for $\mathbf{z} \in S^{1}, \mathbf{x} \in S^{m}, t \in I$, is a homotopy between $f^{\prime}$ and $g^{\prime}$. So, $f$ is homotopic to $g$. 
For the next propositions we need the following maps. Let $\varphi_{1}, \varphi_{2}$, $\varphi: S^{1} \times S^{m} \rightarrow S^{1} \times S^{m}$ be defined as follows. For $\mathbf{u}=(x, y) \in S^{1}$, $\mathbf{v}=(\mathbf{z}, r) \in S^{m}$, where $x, y, r \in D^{1}$ and $\mathbf{z} \in D^{m}$, we define:

$$
\begin{aligned}
& \varphi_{1}(x, y, \mathbf{v})= \begin{cases}(\mathcal{N}(1), \mathbf{v}) & \text { if } y \geq 0, \\
(E(x), \mathbf{v}) & \text { if } y \leq 0,\end{cases} \\
& \varphi_{2}(\mathbf{u}, \mathbf{z}, r)= \begin{cases}(\mathbf{u}, \mathscr{N}(m)) & \text { if } r \geq 0 \\
(\mathbf{u}, E(\mathbf{z})) & \text { if } r \leq 0 .\end{cases}
\end{aligned}
$$

Let $\varphi=\varphi_{1} \circ \varphi_{2}$. It is easy to check that $\varphi=\varphi_{2} \circ \varphi_{1}$, and that $\varphi_{1}, \varphi_{2}$, and $\varphi$ are homotopic to the identity map id: $S^{1} \times S^{m} \rightarrow S^{1} \times S^{m}$. Next, let $A=\varphi^{-1}(\mathscr{N}(1), \mathscr{N}(m))$. Then $A=B \times C$, where $B=$ $\left\{(x, y) \in S^{1} \mid y \geq 0\right\} \subseteq S^{1}$, and $C=\left\{(\mathbf{z}, r) \in S^{m} \mid r \geq 0\right\}$. We see that $B$ is homeomorphic to $D^{1}$ and $C$ is homeomorphic to $D^{m}$. So $A$ is homeomorphic to $D^{m+1}$; let $\theta: A \rightarrow D^{m+1}$ be a homeomorphism. Let $\rho:\left(D^{m+1}, \partial D^{m+1}\right) \rightarrow\left(S^{m}, \mathscr{N}(m)\right)$ represent a nonzero element of $\pi_{m+1}\left(S^{m}\right)$. For a given $f: S^{1} \times S^{m} \rightarrow S^{m}$, let $\Psi(f): S^{1} \times S^{m} \rightarrow S^{m}$ be defined by:

$$
\Psi(f)(x)= \begin{cases}f \circ \varphi(x) & \text { for } x \in\left(S^{1} \times S^{m} \backslash A\right) \cup \partial A, \\ \rho \circ \theta(x) & \text { for } x \in A .\end{cases}
$$

Proposition 1.9. Let $\varphi, A, f, \Psi(f)$ be as above, and let $m \geq 3$. Then, $f$ is homotopic to $f \circ \varphi, \operatorname{deg}_{1}(f)=\operatorname{deg}_{1}(f \circ \varphi)=\operatorname{deg}_{1}(\Psi(f))$, and $\operatorname{deg}_{2}(f)=\operatorname{deg}_{2}(f \circ \varphi)=\operatorname{deg}_{2}(\Psi(f))+1$, where the addition is in $\pi_{m+1}\left(S^{m}\right)=\mathbb{Z}_{2}$.

Proof. Since $\varphi$ is homotopic to the identity, and $\Psi(f)$ coincides with $f \circ \varphi$ on half of $S^{1} \times S^{m}$, it follows that: $f$ is homotopic to $f \circ \varphi$; they have the same $\operatorname{deg}_{1}$ and $\operatorname{deg}_{2}$; and $\operatorname{deg}_{1}(f)=\operatorname{deg}_{1}(\Psi(f))$. Now, by Proposition 1.5, we have that $\operatorname{deg}_{2}(f \circ \varphi)=[F(f \circ \varphi)]$. The inverse image $F(f \circ \varphi)^{-1}(\mathscr{N}(m))$ contains the set

$$
\begin{aligned}
D & =\left\{(x, y, \mathbf{z}, r) \in S^{m+1} \mid x, y, r \in D^{1}, \mathbf{z} \in D^{m-1}, y \geq 0, r \geq 0\right\} \\
& \subseteq S^{m+1},
\end{aligned}
$$

and $F(f \circ \varphi)(\partial D)=F(\Psi(f))(\partial D)=\mathscr{N}(m)$. The facts that: $D$ is homeomorphic to $D^{m+1} ; F(f \circ \varphi)(D)=\mathscr{N}(m)$; the restriction of $\Psi(f)$ on $A$ gives a nontrivial element of $\pi_{m+1}\left(S^{m}\right)$; and $\Psi(f)$ is equal to $f \circ \varphi$ on $S^{1} \times S^{m} \backslash A$; imply that the restriction of $F(\Psi(f))$ to $D$ gives a nontrivial element of $\pi_{m+1}\left(S^{m}\right)$, and that $[F(f \circ \varphi)]=$ $[F(\Psi(f))]+1$. Hence $\operatorname{deg}_{2}(f)=\operatorname{deg}_{2}(\Psi(f))+1$. 
Proposition 1.10. Let $f, g: S^{1} \times S^{m} \rightarrow S^{m}$ be two homotopic maps, $m \geq 3$, and $\Psi(f), \Psi(g)$ be defined by (1.8). Then $\Psi(f)$ is homotopic to $\Psi(g)$.

Proof. W.l.o.g. we can assume that

$$
f(\mathcal{N}(1), \mathscr{N}(m))=g(\mathscr{N}(1), \mathcal{N}(m))=\mathscr{N}(m) .
$$

Since $m \geq 2$, by the homotopy extension theorem, we can homotope a given homotopy from $f$ to $g$ to a homotopy $H: S^{1} \times S^{m} \times I \rightarrow$ $S^{m}, \operatorname{rel}(\mathscr{N}(1), \mathscr{N}(m))$, i.e. such that $H(\mathscr{N}(1), \mathscr{N}(m), t)=\mathscr{N}(m)$ for every $t \in I$. By the definition of $\varphi$, it follows that $H \circ \varphi$ is a homotopy from $f \circ \varphi$ to $g \circ \varphi \operatorname{rel} A$, and moreover $\operatorname{rel} \partial A$. Now, $H^{\prime}: S^{1} \times S^{m} \times I \rightarrow S^{m}$ defined by: $H^{\prime}(x, t)=H \circ \varphi(x, t)$ for $x \in$ $\left(S^{1} \times S^{m} \backslash A\right) \cup \partial A$; and $H^{\prime}(x, t)=\rho \circ \theta(x)$ for $x \in A$; is a homotopy from $\Psi(f)$ to $\Psi(g)$.

Proposition 1.11. Let $f: S^{1} \times S^{m} \rightarrow S^{m}$ be given, and $m \geq 3$. Let $\Psi(f)$ and $\Psi(\Psi(f))$ be defined by (1.8). Then $\Psi(\Psi(f))$ is homotopic to $f$.

Proof. In the definition of $\Psi(f)$ we have used

$$
A=\varphi^{-1}(\mathcal{N}(1), \mathcal{N}(m)) .
$$

Let $A^{\prime}$ be any subset of $A$ with a homeomorphism $\theta^{\prime}: A \rightarrow D^{m+1}$ such that $A \backslash$ int $A^{\prime}$ is homeomorphic to $S^{m} \times I$. Then it is easy to check that the map $\Psi^{\prime}(f)$ defined by (1.8) using $A^{\prime}$ instead of $A$, is homotopic to $\Psi(f) \operatorname{rel} S^{1} \times S^{m} \backslash \operatorname{int} A$. Now, let $A_{1}$ be such a subset of $A$ with a homeomorphism $\theta_{1}: A_{1} \rightarrow D^{m+1}$, and let $A_{2}$ be a subset of $\varphi^{-1}(A)$ with a homeomorphism $\theta_{2}: A_{2} \rightarrow D^{m+1}$, such that $A_{2} \cap A=$ $\varnothing$. For such $A_{2}$, the restriction of $\varphi$ to $A_{2}$ is a homeomorphism from $A_{2}$ to $\varphi\left(A_{2}\right)$, and moreover, $\theta_{2} \circ \varphi^{-1}: \varphi\left(A_{2}\right) \rightarrow D^{m+1}$ is a homeomorphism. We define $\Psi(\Psi(f))$ using $A_{1}$ and $\theta_{1}$, and define $\Psi(f)$ using $\varphi\left(A_{2}\right)$ and $\theta_{2} \circ \varphi^{-1}$. Then, it can be checked that:

(a) $\Psi(\Psi(f))(x)=f \circ \varphi \circ \varphi(x)$ for $x \in\left(S^{1} \times S^{m} \backslash A_{1} \backslash A_{2}\right) \cup \partial A_{1} \cup \partial A_{2}$;

(b) $\Psi(\Psi(f))(x)=\rho \circ \theta_{1}(x)$, for $x \in A_{1}$, and

(c) $\Psi(\Psi(f))(x)=\rho \circ \theta_{2}(x)$ for $x \in A_{2}$.

Since $A_{1} \cup A_{2} \subseteq \varphi^{-1}(A)=\varphi^{-1}\left(\varphi^{-1}(\mathscr{N}(1), \mathscr{N}(m))\right)$, it follows that $\varphi^{-1}(A)$ is homeomorphic to an $(m+1)$-ball, and $\rho \circ \theta_{1}$ and $\rho \circ \theta_{2}$ represent nontrivial elements of $\pi_{m+1}\left(S^{m}\right)=\mathbb{Z}_{2}$, it follows that the restriction of $\Psi(\Psi(f))$ to $\varphi^{-1}(A)$ represents the trivial element of 
$\pi_{m+1}\left(S^{m}\right)$. Hence, the restriction of $\Psi(\Psi(f))$ to $\varphi^{-1}(A)$ is homotopic to the constant map $\operatorname{rel} \partial \varphi^{-1}(A)$, which implies that $\Psi(\Psi(f))$ is homotopic to $f \circ \varphi \circ \varphi \operatorname{rel} S^{1} \times S^{m} \backslash$ int $\varphi^{-1}(A)$. Since $f \circ \varphi \circ \varphi\left(\varphi^{-1}(A)\right)=$ $f \circ \varphi(A)=f(\mathscr{N}(1), \mathscr{N}(m))$, it follows that $\Psi(\Psi(f))$ is homotopic to the map $f \circ \varphi \circ \varphi$. On the other hand, $f \circ \varphi \circ \varphi$ is homotopic to $f \circ \varphi$ and $f \circ \varphi$ is homotopic to $f$, i.e. $\Psi(\Psi(f))$ is homotopic to $f$.

THEOREM 1.12. Let $f, g: S^{1} \times S^{m} \rightarrow S^{m}$ be given maps, and $m \geq$ 3. The map $f$ is homotopic to the map $g$, if and only if $\operatorname{deg}_{1}(f)=$ $\operatorname{deg}_{1}(g)$ and $\operatorname{deg}_{2}(f)=\operatorname{deg}_{2}(g)$.

Proof. If $f$ and $g$ are homotopic, then the conclusion follows from Proposition 1.3.

Conversely, let $\operatorname{deg}_{1}(f)=\operatorname{deg}_{1}(g)$ and $\operatorname{deg}_{2}(f)=\operatorname{deg}_{2}(g)$. If $\operatorname{deg}_{2}(f)=0$, then the conclusion follows from Proposition 1.8. If $\operatorname{deg}_{2}(f) \neq 0$, then $\operatorname{deg}_{1}(\Psi(f))=\operatorname{deg}_{1}(\Psi(g))$ and $\operatorname{deg}_{2}(\Psi(f))=$ $\operatorname{deg}_{2}(\Psi(g))=0$, by Proposition 1.9. Now, Proposition 1.8 implies that $\Psi(f)$ is homotopic to $\Psi(g)$, Proposition 1.10 implies that $\Psi(\Psi(f))$ is homotopic to $\Psi(\Psi(g))$, and Proposition 1.11 implies that $f$ is homotopic to $g$.

COROLlARY 1.13. There is a bijection from the set of homotopy classes of maps from $S^{1} \times S^{m}$ to $S^{m}$, to the set $\mathbb{Z} \times \mathbb{Z}_{2}$. In other words, there is a bijection from the cohomotopy group $\pi^{m}\left(S^{1} \times S^{m}\right)$ to $\mathbb{Z} \times \mathbb{Z}_{2}$.

Corollary 1.13 can be proved by homotopy theory methods. Ross Geoghegan has provided a proof of this corollary, in the spirit of a paper by V. L. Hansen [Ha], using Whitehead products, and Peter Hilton has made the remark that degrees 1 and 2 are closely related to the Hopf construction. These homotopy theory methods were not sufficient for the ideas developed in the later parts of this paper, so I have proved all of the properties of degrees 1 and 2 which were needed later.

Proposition 1.14. A map $f: S^{1} \times S^{m} \rightarrow S^{m}$ has an extension $\bar{f}: S^{1} \times D^{m+1} \rightarrow S^{m}$, if and only if $\operatorname{deg}_{1}(f)=0=\operatorname{deg}_{2}(f)$.

Proof. If $f$ has an extension $\bar{f}$, then the restriction of $f$ to $\mathscr{N}(1) \times$ $S^{m}$, has an extension on $\mathscr{N}(1) \times D^{m+1}$, which implies that $\operatorname{deg}_{1}(f)=$ 0 . W.l.o.g. we may assume that $f\left(S^{1} \times\{\mathscr{N}(m)\}\right)=\mathscr{N}(m)$, and then 
$\operatorname{deg}_{2}(f)$ is equal to $[F(f)]$. Now, the extension $\bar{f}$ gives an extension of the map $\bar{F}: S^{m+1} \cup(0,0) \times D^{m} \rightarrow S^{m}$ defined by $F(f)$ on $S^{m+1}$, and by $\bar{F}\left((0,0) \times D^{m}\right)=\mathscr{N}(m)$, where $S^{m+1} \cup(0,0) \times D^{m} \subseteq D^{m+2}$, which shows that $F(f)$ has an extension $\bar{F}(f): D^{m+2} \rightarrow S^{m}$. Hence $[F(f)]=0$, i.e. $\operatorname{deg}_{2}(f)=0$.

Conversely, let $\operatorname{deg}_{1}(f)=0=\operatorname{deg}_{2}(f)$. Then, by Proposition 1.7, $f$ is homotopic to a map $g$, such that $g(\mathbf{z}, \mathbf{x})=h(\mathbf{x})$ for every $\mathbf{z} \in S^{1}, \mathbf{x} \in S^{m}$, where $h: S^{m} \rightarrow S^{m}$, has degree 0 , i.e. $\operatorname{deg}(h)=0$. This implies that $h$ has an extension $\bar{h}: D^{m+1} \rightarrow S^{m}$, and so $g$ has an extension $\bar{g}$ defined by $\bar{g}(\mathbf{z}, \mathbf{y})=\bar{h}(\mathbf{y})$, for every $\mathbf{z} \in S^{1}$ and $\mathbf{y} \in D^{m+1}$. Now, the homotopy from $f$ to $g$ and the extension $\bar{g}$ of $g$ give the required extension of $f$.

At the end of this part we will describe specific maps $h(m): S^{1} \times$ $S^{m} \rightarrow S^{m}, m \geq 3$, with $\operatorname{deg}_{2}(h(m)) \neq 0$, obtained by suspensions, via the Hopf map from $S^{3}$ to $S^{2}$.

If $X$ is a space the suspension $\Sigma X$ of $X$ is the factor space $X \times$ $D^{1} / \alpha$, where $\alpha$ is the equivalence whose classes are:

$$
(x, t)^{\alpha}= \begin{cases}\{(x, t)\} & \text { for } t \neq 1 \text { and } t \neq-1 \\ \{(y, t) \mid y \in X\} & \text { for } t=1 \text { or } t=-1 .\end{cases}
$$

For a given $f: S^{1} \times S^{m} \rightarrow S^{m}$ we define a map $S(f): S^{1} \times S^{m+1} \rightarrow$ $S^{m+1}$ by suspension of $f$ on the second factor, where $\Sigma S^{m}$ is identified with $S^{m+1}$ as a subset of $D^{m+1} \times D^{1}$, i.e.

$$
S(f)\left(\mathbf{x},(\mathbf{y}, t)^{\alpha}\right)=(f(\mathbf{x}, \mathbf{y}), t)^{\alpha} .
$$

THEOREM 1.15. Let $f: S^{1} \times S^{m} \rightarrow S^{m}$ be a given map, $m \geq 3$. Then for $S(f): S^{1} \times S^{m+1} \rightarrow S^{m+1}$ defined as above, $\operatorname{deg}_{1}(f)=$ $\operatorname{deg}_{1}(S(f))$ in $\mathbb{Z}$, and $\operatorname{deg}_{2}(f)=\operatorname{deg}_{2}(S(f))$ in $\mathbb{Z}_{2}$.

Proof. (a) For a point $Q \in S^{1}$, if $h$ is the restriction of $f$ to $\{Q\} \times$ $S^{m}$, then the restriction $H$ of $S(f)$ to $\{Q\} \times \Sigma S^{m}=\{Q\} \times S^{m+1}$ is the suspension of $h$, i.e. $H=\Sigma h$. Since $\Sigma: H_{m}\left(S^{m}\right) \rightarrow H_{m+1}\left(S^{m+1}\right)$ is an isomorphism (see [W]), it follows that $\operatorname{deg}(h)=\operatorname{deg}(\Sigma h)$. Hence $\operatorname{deg}_{1}(f)=\operatorname{deg}_{1}(S(f))$.

(b) If $\operatorname{deg}_{2}(f)=0$, then Proposition 1.7 implies that $f$ is homotopic to $g: S^{1} \times S^{m} \rightarrow S^{m}$ where $g(\mathbf{z}, \mathbf{x})=g(Q, \mathbf{x})$ for a point $Q \in S^{1}$. Then from the definition of $S(g)$ it follows that $S(g)(\mathbf{z}, \mathbf{y})=$ $S(f)(Q, \mathbf{y})$. The homotopy from $f$ to $g$ can be extended to a homotopy from $S(f)$ to $S(g)$. Since $\operatorname{deg}_{2}(S(g))=0$, it follows that $\operatorname{deg}_{2}(S(f))=0$. Hence, $\operatorname{deg}_{2}(f)=0$ implies $\operatorname{deg}_{2}(S(f))=0$. 
(c) From the definition of $S(f)$ it follows that $S(\Psi(f))=\Psi(S(f))$, where $\Psi(f)$ is defined by (1.8). If $\operatorname{deg}_{2}(f)=1$ in $\mathbb{Z}_{2}$, then by Proposition 1.9, $\operatorname{deg}_{2}(\Psi(f))=0$, which implies that $\operatorname{deg}_{2}(S(\Psi(f)))=$ $\operatorname{deg}_{2}(\Psi(S(f)))=0$. Then, again by Proposition 1.9, it follows that $\operatorname{deg}_{2}(S(f))=1$ in $\mathbb{Z}_{2}$. Hence, $\operatorname{deg}_{2}(f)=1$ in $\mathbb{Z}_{2}$, implies $\operatorname{deg}_{2}(S(f))$ $=1$ in $\mathbb{Z}_{2}$.

At the end, the conclusions of (b) and (c) imply that $\operatorname{deg}_{2}(f)=$ $\operatorname{deg}_{2}(S(f))$.

Next, for $m \geq 2$, let $h(m): S^{1} \times S^{m} \rightarrow S^{m}$ be defined by:

$$
h(m)(\mathbf{z}, \mathbf{w}, x)=(\mathbf{z w}, x)
$$

where $\mathbf{z} \in S^{1} \subseteq \mathbb{C},(\mathbf{w}, \mathbf{x}) \in S^{m} \subseteq D^{2} \times D^{m-1}, \mathbf{w} \in D^{2} \subseteq \mathbb{C}, \mathbf{x} \in$ $D^{m-1}, \mathbb{C}$ is the set of complex numbers, and $\mathbf{z w}$ is the product of $\mathbf{z}$ and $\mathbf{w}$ as complex numbers. If we consider $S^{m}$ as a subset of $D^{2} \times D^{m-1}$, and if we identify $\Sigma S^{m}$ with $S^{m+1}$ as a subset of $D^{2} \times$ $D^{m-1} \times D^{1}$, from the definitions of $h(m)$ and $S(f)$, it follows that $S(h(m))=h(m+1)$.

TheOREM 1.16. For $m \geq 3, \operatorname{deg}_{1}(h(m))=1$ in $\mathbb{Z}$, and $\operatorname{deg}_{2}(h(m))$ $=1$ in $\mathbb{Z}_{2}$.

Proof. From the definition of $h(3)$, it follows that the restriction of $h(3)$ to $\{\mathbf{z}\} \times S^{3}$ for a point $\mathbf{z} \in S^{1}$ is a rotation on the first two coordinates of $S^{3}$, with the angle of rotation obtained from $\mathbf{z}$. Hence, the degree of the restriction of $h(3)$ to $\{z\} \times S^{3}$ is 1 . Then, by induction, Theorem 1.15, implies that $\operatorname{deg}_{1}(h(m))=1$ for $m \geq 3$.

The map $h(2)$ has the property that $h(2)(\mathbf{z}, \mathscr{N}(2))=\mathscr{N}(2)$ for each $\mathbf{z} \in S^{1}$. We define $\overline{h(2)}: X \rightarrow S^{2}$ by: $\overline{h(2)}$ restricted to $S^{1} \times S^{2}$ is $h(2)$, and $\overline{h(2)}\left(D^{2}\right)=\mathscr{N}(2)$, where $X=S^{1} \times S^{2} \cup D^{2}$ with the identification of $S^{1} \times\{\mathscr{N}(2)\}$ with $\partial D^{2}$. Then, it can be checked that $\overline{h(2)} \circ K: S^{3} \rightarrow S^{2}$ is the Hopf map, and that $\overline{h(3)} \circ K: S^{4} \rightarrow S^{3}$ is homotopic to the suspension $\Sigma(\overline{h(2)} \circ K)$. Since $[\overline{h(2)} \circ K]$ is a generator of $\pi_{3}\left(S^{2}\right)$ and the suspension $\Sigma: \pi_{3}\left(S^{2}\right) \rightarrow \pi_{4}\left(S^{3}\right)$ is an epimorphism (see [W]), it follows that $\operatorname{deg}_{2}(h(3))=1$ in $\mathbb{Z}_{2}$. Then, by induction, Theorem 1.15, implies that $\operatorname{deg}_{2}(h(m))=1$ for $m \geq$ 3 .

II. Standard models. In this section we will define several standard models and prove several facts about extensions of maps.

Let $S E: S^{1} \times D^{m} \rightarrow \mathbb{R}^{m+1}$ be the embedding defined by: (2.1) $S E(\mathbf{z}, r, \mathbf{y})=((2-r) \mathbf{z}, \mathbf{y}), \quad$ for $\mathbf{z} \in S^{1},(r, \mathbf{y}) \in D^{m}, r \in D^{1}$. 

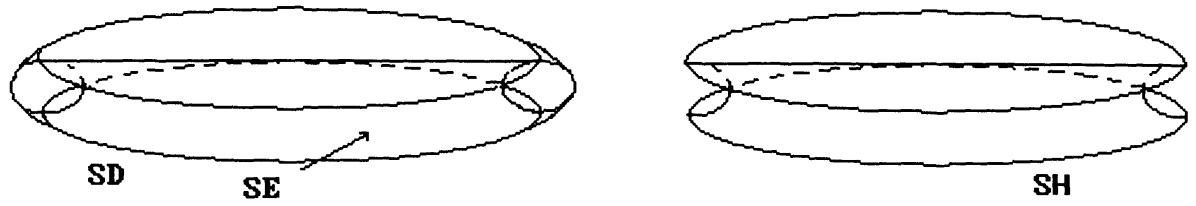

FIGURE 2.1

We say that $S E$ is the standard embedding. It follows from the definition of $S E$ that $S E$ is orientation preserving, where $S^{1} \times D^{m}$ has the product orientation of the standard orientations, and the restriction of $S E$ from $\mathscr{N}(1) \times D^{m}$ to $\{0\} \times \mathbb{R}^{m}$ is orientation reversing. We will use the same notation $S E$ for the image of the map $S E$. In other words,

$S E=\left\{\left.(\mathbf{z}, \mathbf{x})\left|\mathbf{z} \in \mathbb{R}^{2}, \quad \mathbf{x} \in \mathbb{R}^{m-1}, \quad 1 \leq\right| \mathbf{z}|\leq 3,| \mathbf{x}\right|^{2} \leq 1-(|\mathbf{z}|-2)^{2}\right\}$.

Let $S D$ be the subset of $\mathbb{R}^{m+1}$ defined by:

$$
S D=S E \cup\left\{(\mathbf{z}, \mathbf{x})\left|\mathbf{z} \in \mathbb{R}^{2}, \mathbf{x} \in D^{m-1},\right| \mathbf{z} \mid \leq 2\right\} .
$$

Let $S H=\operatorname{cl}(S D \backslash S E)$. Then $S D=S E \cup S H$. We say that $S D$ is the standard model for $D^{2}$ in $\mathbb{R}^{m+1}$, because $S D$ is a 1-regular neighborhood of the disk $\left\{(\mathbf{z}, \mathbf{0}) \in \mathbb{R}^{m+1}\left|\mathbf{z} \in \mathbb{R}^{2},\right| \mathbf{z} \mid \leq 2\right\}$ in $\mathbb{R}^{m+1}$. The standard model $S D$ is homeomorphic to $D^{m+1}$. A schematic picture for $S D, S H$ and $S E$ in the case $m=2$, is shown in Figure 2.1. We use the notation $\partial S D$ for the homeomorphism $\partial S D: S^{m} \rightarrow$ $\partial S D$ defined as follows. For $(\mathbf{z}, \mathbf{x}) \in S^{m}, \mathbf{z} \in D^{2}, \mathbf{x} \in D^{m-1}$ :

$$
\partial S D(\mathbf{z}, \mathbf{x})= \begin{cases}\left(4 \cdot \mathbf{z}, \frac{\mathbf{x}}{|\mathbf{x}|}\right), & |\mathbf{z}| \leq \frac{1}{2} \\ \left((2|\mathbf{z}|+1) \cdot \frac{\mathbf{z}}{|\mathbf{z}|}, \sqrt{1-(2|\mathbf{z}|-1)^{2}} \cdot \frac{\mathbf{x}}{|\mathbf{x}|}\right), & \frac{1}{2} \leq|\mathbf{z}| .\end{cases}
$$

The set $S H$ is also homeomorphic to $D^{m+1}$, and we denote by $\partial S H$ the homeomorphism $\partial S H: S^{m} \rightarrow \partial S H$ defined as follows. For $(\mathbf{z}, \mathbf{x}) \in S^{m}, \mathbf{z} \in D^{2}, \mathbf{x} \in D^{m-1}$ : (2.5)

$$
\partial S H(\mathbf{z}, \mathbf{x})= \begin{cases}\left(4 \cdot \mathbf{z}, \frac{\mathbf{x}}{\mid \mathbf{x}}\right), & |\mathbf{z}| \leq \frac{1}{2} \\ \left((3-2|\mathbf{z}|) \cdot \frac{\mathbf{z}}{|\mathbf{z}|}, \sqrt{1-(2|\mathbf{z}|-1)^{2}} \cdot \frac{\mathbf{x}}{|\mathbf{x}|}\right), & \frac{1}{2} \leq|\mathbf{z}| .\end{cases}
$$

Let $\varphi: S H \cup \partial S E \rightarrow S^{m-1}$ be a given map. Let $\psi=\varphi \mid \partial S D$ and $f=\varphi \mid \partial S E$ be the restrictions of $\varphi$ to $\partial S D$ and $\partial S E$. Let $\partial S E$ be the restriction of the map $S E$ to $\partial\left(S^{1} \times D^{m}\right)=S^{1} \times S^{m-1}$, i.e. $\partial S E: S^{1} \times S^{m-1} \rightarrow \partial S E$. 


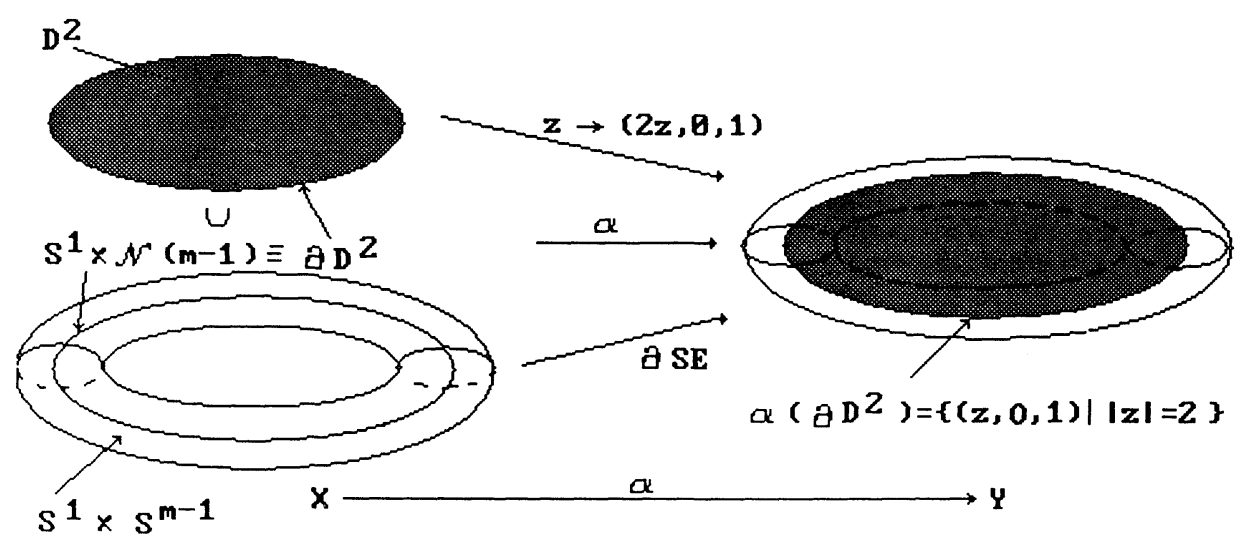

Figure 2.2

Proposition 2.1. Let $\varphi, f, \psi$ be as above. Then, for $m \geq 4, \psi$ has an extension $\xi: S D \rightarrow S^{m-1}$ if and only if $\operatorname{deg}_{2}(f \circ \partial S E)=0$.

Proof. Because $\pi_{1}\left(S^{m-1}\right)=0=\pi_{2}\left(S^{m-1}\right)$, for $m \geq 4$, using the homotopy extension theorem (HET), the map $\varphi$ is homotopic to a map $\varphi: \partial S E \cup S H \rightarrow S^{m-1}$, such that $\varphi^{\prime}(\mathbf{z}, \mathbf{0}, 1)=\mathcal{N}(m-1)$, for every $\mathbf{z} \in \mathbb{R}^{2}$ with $|\mathbf{z}| \leq 2$. Let $f^{\prime}$ be the restriction of $\varphi^{\prime}$ to $\partial S E$, and $\psi^{\prime}$ be the restriction of $\varphi^{\prime}$ to $\partial S D$. Then $f \circ \partial S E$ is homotopic to $f^{\prime} \circ \partial S E$, and $\psi$ is homotopic to $\psi^{\prime}$. The set, $Y=\partial S E \cup$ $\left\{(\mathbf{z}, \mathbf{0}, 1) \in \mathbb{R}^{2} \times \mathbb{R}^{m-2} \times \mathbb{R}|| \mathbf{z} \mid \leq 2\right\}$ is homeomorphic to the factor space $X$ obtained from $S^{1} \times S^{m-1} \cup D^{2}$, and one homeomorphism $\alpha: X \rightarrow Y$ is defined by: $\alpha(\mathbf{z})=(2 \mathbf{z}, \mathbf{0}, 1)$ for $\mathbf{z} \in D^{2}$, and $\alpha \mid S^{1} \times$ $S^{m-1}=\partial S E$. Figure 2.2, illustrates the spaces $X$ and $Y$ and the homeomorphism $\alpha$. Next, $\alpha(X)$ is a deformation retract of $\partial S E \cup$ $S H$, with a homotopy $G:(\partial S E \cup S H) \times I \rightarrow \partial S E \cup S H$, such that the end of the homotpoy $G_{1}$ satisfies: $G_{1} \circ \partial S D=\alpha \circ K$, where $K=K_{1}$ is defined by (1.2). Now let $\varphi^{\prime \prime}=\varphi^{\prime} \circ G_{1}$, let $f^{\prime \prime}$ be the restriction of $\varphi^{\prime \prime}$ to $\partial S E$ and let $\psi^{\prime \prime}$ be the restriction of $\varphi^{\prime \prime}$ to $\partial S D$. With the above notation and homotopies, we have that: $\psi$ has an extension on $S D$ iff $\psi^{\prime \prime}$ has an extension iff $\psi^{\prime \prime} \circ \partial S D$ has an extension iff the element $\left[\psi^{\prime \prime} \circ \partial S D\right]=0$ in $\pi_{m}\left(S^{m-1}\right)$ iff $\left[\varphi^{\prime \prime} \circ \partial S D\right]=0$ in $\pi_{m}\left(S^{m-1}\right)$ iff $\left[\varphi^{\prime} \circ G_{1} \circ \partial S D\right]=0$ in $\pi_{m}\left(S^{m-1}\right)$ iff $\left[\varphi^{\prime} \circ \alpha \circ K\right]=0$ in $\pi_{m}\left(S^{m-1}\right)$ iff $\operatorname{deg}_{2}\left(\varphi^{\prime} \circ \partial S E\right)=0$ iff $\operatorname{deg}_{2}(\varphi \circ \partial S E)=0$ iff $\operatorname{deg}_{2}(f \circ \partial S E)$ $=0$.

Let $A N$ be the annulus in $\mathbb{R}^{2} \subseteq \mathbb{R}^{m+1}$ defined by

$$
A N=\left\{(\mathbf{z}, \mathbf{0}) \in \mathbb{R}^{m+1}\left|\mathbf{z} \in \mathbb{R}^{2}, 2 \leq\right| \mathbf{z} \mid \leq 5\right\} .
$$


Let $D_{1}=\left\{(\mathbf{z}, \mathbf{0}) \in \mathbb{R}^{m+1}\left|\mathbf{z} \in \mathbb{R}^{2},\right| \mathbf{z} \mid \leq 5\right\}, D_{2}=\left\{(\mathbf{z}, \mathbf{0}) \in \mathbb{R}^{m+1} \mid \mathbf{z} \in\right.$ $\left.\mathbb{R}^{2},|\mathbf{z}| \leq 2\right\}$, and $C_{j}=\partial D_{j}$, be the boundary circles of $A N$. Let $S A N$ be the 1-regular neighborhood of $A N$ in $\mathbb{R}^{m+1}$, and let $S C_{j}$ be the 1-regular neighborhood of $C_{j}$ in $\mathbb{R}^{m+1}$, for $j=1,2$. Note that $S C_{2}=S E$, and

$S C_{1}=\left\{\left.(\mathbf{z}, \mathbf{x})\left|\mathbf{z} \in \mathbb{R}^{2}, \quad \mathbf{x} \in \mathbb{R}^{m-1}, 4 \leq\right| \mathbf{z}|\leq 6,| \mathbf{x}\right|^{2} \leq 1-(|\mathbf{z}|-5)^{2}\right\}$.

We orient the circles $C_{j}$ by the induced orientation from the disks $D_{j}$ where $D_{j}$ are oriented by the induced orientation from $\mathbb{R}^{2}$. In the same way as the definition of the standard embedding $S E$, i.e. $S C_{2}$, we can define a standard embedding $S C_{1}: S^{1} \times D^{m} \rightarrow \mathbb{R}^{m+1}$, such that it is isotopic to the standard embedding $S E$, it is orientation preserving, preserves the orientations from $S^{1} \times\{\boldsymbol{0}\}$ to $C_{1}$ and the restriction of $S C_{1}$ from $\mathscr{N}(1) \times D^{m}$ to $\{0\} \times \mathbb{R}^{m}$ is orientation reversing. Let $S C=S C_{1} \cup S C_{2}$, and let $S A H=\operatorname{cl}(S A N \backslash S C)$. Then $S A N=$ $S C \cup S A H$. The model $S A N$ is homeomorphic to $S^{1} \times D^{m}$. We use the notation $S A N$ for the homeomorphism $S A N: S^{1} \times D^{m} \rightarrow S A N$ defined as follows. For $(\mathbf{z}, r, \mathbf{x}) \in S^{1} \times D^{m}, \mathbf{z} \in S^{1},(r, \mathbf{x}) \in D^{m}$, $r \in D^{1}, \mathbf{x} \in D^{m-1}$ :

(2.8) $S A N(\mathbf{z}, r, \mathbf{x})=\left\{\begin{array}{lc}((4-2 r) \cdot \mathbf{z}, A \cdot \mathbf{x}), & -1 \leq r \leq-\frac{1}{2}, \\ \left(\left(\frac{7}{2}-3 r\right) \cdot \mathbf{z}, B \cdot \mathbf{x}\right), & -\frac{1}{2} \leq r \leq \frac{1}{2}, \\ ((3-2 r) \cdot \mathbf{z}, C \cdot \mathbf{x}), & \frac{1}{2} \leq r \leq 1,\end{array}\right.$

where: $A=0$ for $r=-1, C=0$ for $r=1$, and

$$
\begin{aligned}
& A=\frac{\sqrt{1-(2 r+1)^{2}}}{\sqrt{1-r^{2}}}, \quad B=\frac{1}{\sqrt{1-r^{2}}}, \quad \text { and } \quad C=\frac{\sqrt{1-(2 r-1)^{2}}}{\sqrt{1-r^{2}}}, \\
& \text { for } r \neq \pm 1 \text {. }
\end{aligned}
$$

We denote the restriction of $S C_{j}$ and $S A N$ to the boundaries by $\partial S C_{j}$ and $\partial S A N$, i.e. $\partial S C_{j}: S^{1} \times S^{m-1} \rightarrow \partial S C_{j}$ and $\partial S A N: S^{1} \times$ $S^{m-1} \rightarrow \partial S A N$.

The set $S A H$ is also homeomorphic to $S^{1} \times D^{m}$.

Proposition 2.2. Let $\varphi: S A H \cup \partial S C \rightarrow S^{m-1}$ be given and let $f_{j}=\varphi \mid \partial S C_{j}, j=1,2$, and $\psi=\varphi \mid \partial S A N$ be the restriction of $\varphi$ to $\partial S C_{j}$ and $\partial S A N$, respectively. Then

$\operatorname{deg}_{i}(\psi \circ \partial S A N)=\operatorname{deg}_{i}\left(f_{1} \circ \partial S C_{1}\right)+\operatorname{deg}_{i}\left(f_{2} \circ \partial S C_{2}\right), \quad$ for $i=1,2$.

Proof. Because $\pi_{1}\left(S^{m-1}\right)=0=\pi_{2}\left(S^{m-1}\right)$ and $S A H$ is homeomorphic to $S^{1} \times D^{m}$, the $H E T$ implies that $\varphi$ is homotopic to a 

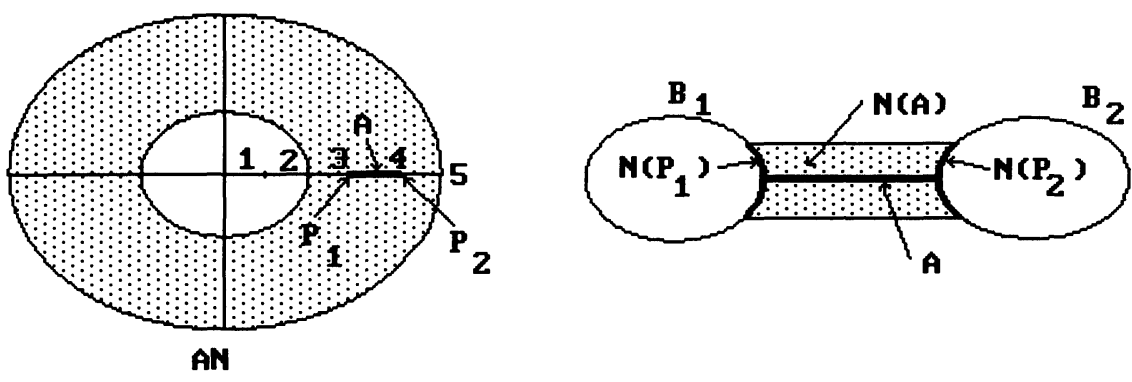

FIGURE 2.3

map $\varphi^{\prime}$, such that $\varphi^{\prime}(S A H)=\mathscr{N}(m-1)$. Let $\psi^{\prime}=\varphi^{\prime} \mid \partial S A N$ and $f_{j}^{\prime}=\varphi^{\prime} \mid \partial S C_{j}$.

Step 1. Let $A$ be the arc $\{((0, r), \mathbf{0}) \mid 3 \leq r \leq 4\}$ in the annulus $A N$, and let $P_{j}, j=1,2$, be its end points, i.e. $P_{1}=\{(0,3, \mathbf{0})\}$ and $P_{2}=$ $\{(0,4,0)\}$. Let $B_{j}=\partial S C_{j} \cap\{0\} \times \mathbb{R}^{m}$, and let $\left(N(A) ; N\left(P_{1}\right), N\left(P_{2}\right)\right)$ be a regular neighborhood triple of $\left(A ; P_{1}, P_{2}\right)$ in $(S A H \cap\{0\} \times$ $\left.\mathbb{R}^{m} ; B_{1}, B_{2}\right)$, schematically shown in Figure 2.3 . Then $\left(N(A) ; N\left(P_{1}\right)\right.$, $\left.N\left(P_{2}\right)\right)$ is triplewise homeomorphic to $\left(D^{m-1} \times I ; D^{m-1} \times\{0\}, D^{m-1} \times\right.$ $\{1\})$, and $N\left(P_{j}\right)$ is an $(m-1)$-ball in $B_{j}, j=1,2$.

We fix a product structure $N(A)=B_{1} \times I$ on $N(A)$ via the homeomorphism $S A N$. We orient $N(A)$ by the induced orientation from $\{0\} \times \mathbb{R}^{m}$, and $N\left(P_{j}\right)$ by the induced orientation from $\partial N(A)$, and define a map $g: \partial S C_{1} \cap\{0\} \times \mathbb{R}^{m} \rightarrow S^{m-1}$, such that $g \mid B_{1} \backslash N\left(P_{1}\right)=$ $f_{1}^{\prime} \mid B_{1} \backslash N\left(P_{1}\right)$, and $\operatorname{deg}(g)=0$. Such $g$ exists because $f^{\prime}\left(N\left(P_{1}\right)\right)=$ $\mathscr{N}(m-1)$. Using the product structure of $N(A)$, we extend the map $g \mid N\left(P_{1}\right)$ to a map $\xi: N(A) \rightarrow S^{m-1}$, by $\xi(x, t)=g(x)$. Let $B$ be the subset of $S A H$ defined by $B=S A N\left(S^{1} \times S A N^{-1}(N(A))\right)$. Using the product structure $S^{1} \times N(A)$ of $B$ via the homeomorphism $S A N$, we can extend the map $\xi$ to a map $\xi^{\prime}: B \rightarrow S^{m-1}$ by $\xi^{\prime}(\mathbf{z}, x)=$ $\xi(x)$. Now, we define a map $\varphi^{\prime \prime}: \partial S C \cup S A H \rightarrow S^{m-1}$ by $\varphi^{\prime \prime}\left|(\partial S C \cup S A H) \backslash B=\varphi^{\prime}\right|(\partial S C \cup S A H) \backslash B$ and $\varphi^{\prime \prime} \mid B=\xi^{\prime}$. Let $\varphi^{\prime \prime} \mid \partial S C_{j}=g_{j}$, and $\varphi^{\prime \prime} \mid \partial S A N=\psi^{\prime \prime}$. Then $\psi^{\prime \prime}=\psi^{\prime}$, and with the above conventions about the orientations we have that $\operatorname{deg}_{1}\left(g_{1} \circ \partial S C_{1}\right)=0$, and $\operatorname{deg}_{1}\left(g_{2} \circ \partial S C_{2}\right)=\operatorname{deg}_{1}\left(f_{1} \circ \partial S C_{1}\right)+$ $\operatorname{deg}_{1}\left(f_{2} \circ \partial S C_{2}\right)$. Moreover, since in the definition of $\varphi^{\prime \prime}$ we have used the product structure on $B$, Proposition 1.7 implies that $\operatorname{deg}_{2}\left(g_{j} \circ \partial S C_{j}\right)=\operatorname{deg}_{2}\left(f_{j} \circ \partial S C_{j}\right), j=1,2$.

Step 2. Let $\left(K(A) ; K\left(P_{1}\right), K\left(P_{2}\right)\right)$ be a regular neighborhood triple of $\left(A ; P_{1}, P_{2}\right)$ in $\left(S A H ; \partial S C_{1}, \partial S C_{2}\right)$. Then $K\left(P_{j}\right)$ is an $m$-ball 
in $\partial S C_{j}$, and $\left(K(A) ; K\left(P_{1}\right), K\left(P_{2}\right)\right)$ is triplewise homeomorphic to $\left(D^{m} \times I ; D^{m} \times\{0\}, D^{m} \times\{1\}\right)$. We fix a product structure $K(A)=$ $K\left(P_{1}\right) \times I$ on $N(A)$ via the homeomorphism $S A N$. We define a map $h: \partial S C_{1} \rightarrow S^{m-1}$, such that $h\left|\partial S C_{1} \backslash K\left(P_{1}\right)=g_{1}\right| \partial S C_{1} \backslash K\left(P_{1}\right)$, and $h$ restricted to $K\left(P_{1}\right)$ gives the element $\operatorname{deg}_{2}\left(f_{1} \circ \partial S C_{1}\right)$ in $\pi_{m}\left(S^{m-1}\right)$. Using the product structure of $K(A)$, we extend the map $h \mid K\left(P_{1}\right)$ to a map $\mu: K(A) \rightarrow S^{m-1}$, by $\mu(x, t)=h(x)$. Next, let $\varphi^{\prime \prime \prime}: \partial S C \cup S A H \rightarrow S^{m-1}$ be defined by: $\varphi^{\prime \prime \prime} \mid K(A)=\mu$ and $\varphi^{\prime \prime \prime}\left|(\partial S C \cup S A H) \backslash K(A)=\varphi^{\prime \prime}\right|(\partial S C \cup S A H) \backslash K(A)$. Let $\varphi^{\prime \prime \prime} \mid \partial S C_{j}=g_{j}^{\prime}$, and $\varphi^{\prime \prime \prime} \mid \partial S A N=\psi^{\prime \prime \prime}$. Then $\psi^{\prime \prime \prime}=\psi^{\prime \prime}=\psi^{\prime}$, and with the above notation, we have that $\operatorname{deg}_{1}\left(g_{j}^{\prime} \circ \partial S C_{j}\right)=\operatorname{deg}_{1}\left(g_{j} \circ \partial S C_{j}\right)$, $\operatorname{deg}_{2}\left(g_{1}^{\prime} \circ \partial S C_{1}\right)=0$, and $\operatorname{deg}_{2}\left(g_{2}^{\prime} \circ \partial S C_{2}\right)=\operatorname{deg}_{2}\left(g_{1} \circ \partial S C_{1}\right)+$ $\operatorname{deg}_{2}\left(g_{2} \circ \partial S C_{2}\right)$.

The facts that $\operatorname{deg}_{i}\left(g_{1}^{\prime}\right)=0, i=1,2$, and Proposition 1.14 imply that $g_{1}^{\prime}$ has an extension $\bar{g}_{1}^{\prime}: S C_{1} \rightarrow S^{m-1}$, which gives an extension of $\psi^{\prime \prime \prime}$ to a $\psi_{1}: S A H \cup S C_{1} \cup \partial S C_{2} \rightarrow S^{m-1}$. This extension gives a homotopy between $\psi^{\prime \prime \prime} \circ \partial S A N$ and $g_{2}^{\prime} \circ \partial S C_{2}$, which shows that

$$
\begin{aligned}
\operatorname{deg}_{i}(\psi \circ \partial S A N) & =\operatorname{deg}_{i}\left(\psi^{\prime \prime} \circ \partial S N\right)=\operatorname{deg}_{i}\left(g_{2}^{\prime} \circ \partial S C_{2}\right) \\
& =\operatorname{deg}_{i}\left(f_{1} \circ \partial S C_{1}\right)+\operatorname{deg}_{i}\left(f_{2} \circ \partial S C_{2}\right) .
\end{aligned}
$$

The following corollary follows directly from Propositions 2.2 and 1.14 .

Corollary 2.3. Let $\varphi, f_{j}, \psi$ be as in Proposition 2.2 and let $m \geq 4$. Then, $\psi$ has an extension $\xi: S A N \rightarrow S^{m-1}$ if and only if $\operatorname{deg}_{i}\left(f_{1} \circ \partial S C_{1}\right)+\operatorname{deg}_{i}\left(f_{2} \circ \partial S C_{2}\right)=0, i=1,2$.

Let $\mathbf{4}=(0,4) \in \mathbb{R}^{2}$, and let $K G \subseteq \mathbb{R}^{2} \subseteq \mathbb{R}^{m+1}$ be the disk with two holes defined by:

(2.9) $K G=\left\{(\mathbf{z}, \mathbf{0}) \in \mathbb{R}^{m+1}\left|\mathbf{z} \in \mathbb{R}^{2},\right| \mathbf{z}|\leq 9,| \mathbf{z}-4|\geq 2,| \mathbf{z}+4 \mid \geq 2\right\}$.

Let $D_{1}=\{(\mathbf{z}, \mathbf{0})|| \mathbf{z} \mid \leq 9\}, D_{2}=\{(\mathbf{z}, \mathbf{0})|| \mathbf{z}-\mathbf{4} \mid \leq 2\}, D_{3}=\{(\mathbf{z}, \mathbf{0}) \mid$ $|\mathbf{z}+4| \leq 2\}$ and let $K_{1}=\partial D_{1}, K_{2}=\partial D_{2}, K_{3}=\partial D_{3}$ be the boundary circles of $K G$. Let $S K G$ be the 1-regular neighborhood of $K G$ in $\mathbb{R}^{m+1}$, and let $S K_{j}$ be the 1-regular neighborhood of $K_{j}$ in $\mathbb{R}^{m+1}$, for $j=1,2,3$. We orient the circles $K_{j}$ by the induced orientation from the standard disks $D_{j}$ they bound, where $D_{j}$ are oriented by the induced orientation from $\mathbb{R}^{2}$. In the same way as the definition of the standard embedding $S E$, for each $1 \leq j \leq 3$ we 

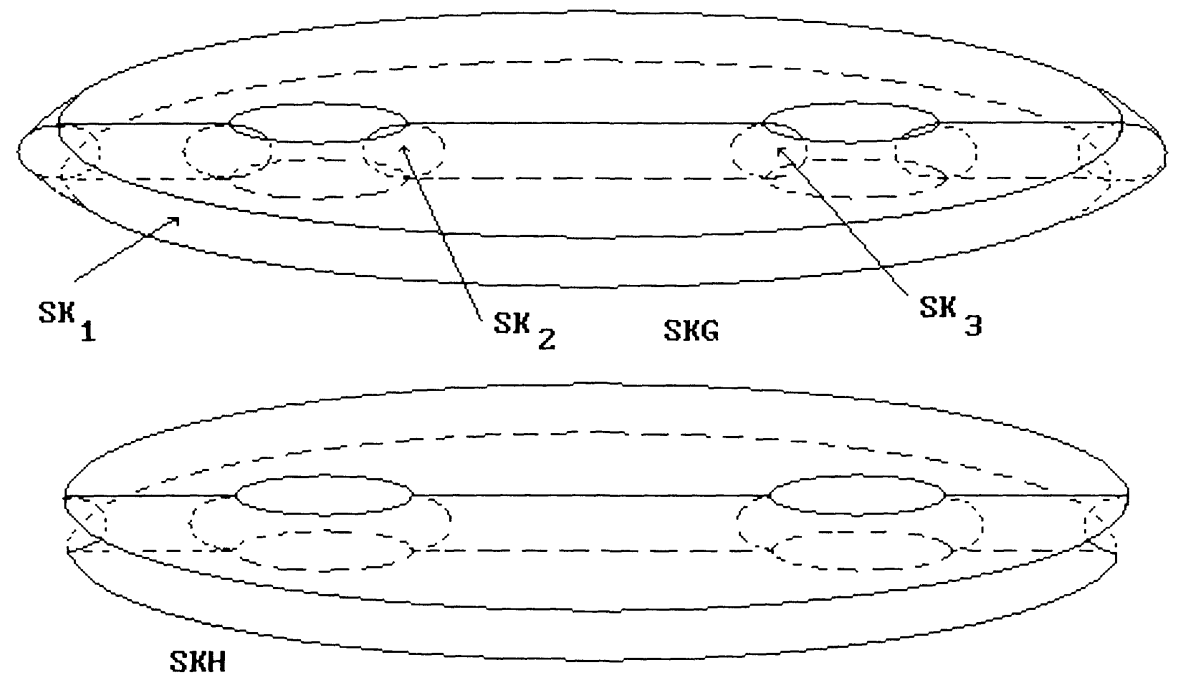

FIGURE 2.4

can define a standard embedding $S K_{j}: S^{1} \times D^{m} \rightarrow \mathbb{R}^{m+1}$, such that they are isotopic to the standard embedding $S E$, they are orientation preserving, and preserve the orientation from $S^{1} \times\{\mathbf{0}\}$ to $K_{j}$. Let $S K=S K_{1} \cup S K_{2} \cup S K_{3}$, and let $S K H=\operatorname{cl}(S K G \backslash S K)$.

We denote the restriction of $S K_{j}$ to the boundaries by $\partial S K_{j}$, i.e. $\partial S K_{j}: S^{1} \times S^{m-1} \rightarrow \partial S K_{j}$.

Proposition 2.4. Let $\varphi: \partial S K \cup S K H \rightarrow S^{m-1}$ be given, and let $\psi=\varphi \mid \partial S K G$ and $f_{j}=\varphi \mid \partial S K_{j}$ be its restrictions to $\partial S K G$ and $\partial S K_{j}$, and let $m \geq 4$. Then, $\psi$ has an extension $\xi: S K G \rightarrow S^{m-1}$ if and only if

(1) $\operatorname{deg}_{1}\left(f_{1} \circ \partial S K_{1}\right)=-\operatorname{deg}_{1}\left(f_{j} \circ \partial S K_{j}\right)$ for $j=2,3$; and

(2) $\operatorname{deg}_{2}\left(f_{1} \circ \partial S K_{1}\right)+\operatorname{deg}_{2}\left(f_{2} \circ \partial S K_{2}\right)+\operatorname{deg}_{2}\left(f_{3} \circ \partial S K_{3}\right)=0$.

Proof. (A) Because $\pi_{1}\left(S^{m-1}\right)=0=\pi_{2}\left(S^{m-1}\right)$, for $m \geq 4$, and $S K H$ is homeomorphic to $K G \times D^{m-1}$, by the $H E T$, the map $\varphi$ is homotopic to a map $\varphi^{\prime}: \partial S K \cup S K H \rightarrow S^{m-1}$, such that $\varphi^{\prime}(S K H)=$ $\mathscr{N}(m-1)$. Let $\psi^{\prime}$ be the restriction of $\varphi^{\prime}$ on $\partial S K G$, and let $f_{j}^{\prime}$ be the restrictions of $\varphi^{\prime}$ on $\partial S K_{j}$. Then $f_{j} \circ \partial S K_{j}$ is homotopic to $f_{j}^{\prime} \circ \partial S K_{j}$ for each $j=1,2,3$, and $\psi$ is homotopic to $\psi^{\prime}$.

(B) If $\operatorname{deg}_{1}\left(f_{1} \circ \partial S K_{1}\right)+\operatorname{deg}_{1}\left(f_{j} \circ S K_{j}\right)=0$ for $j=2,3$, then by applying a variation of the method in Step 1 from the proof of Proposition 2.3 we can accomplish the following: $\operatorname{deg}_{1}\left(f_{j}^{\prime} \circ \partial S K_{j}\right)=0$ 


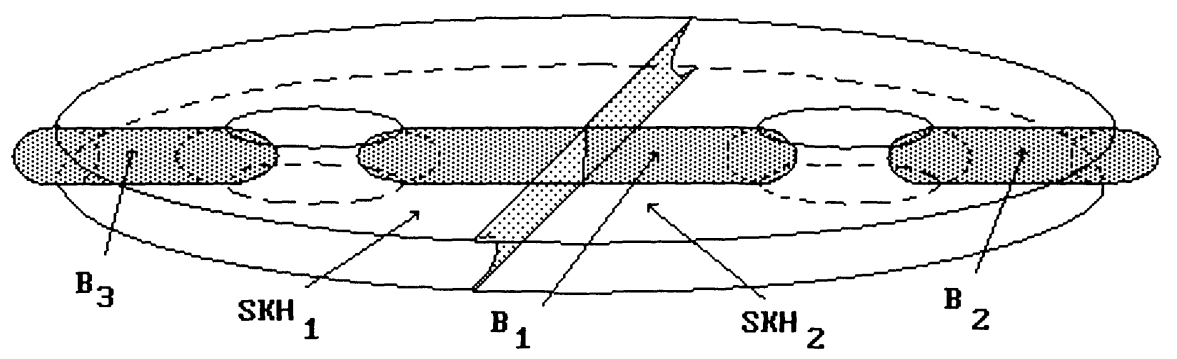

Figure 2.5

and $\operatorname{deg}_{2}\left(f_{j}^{\prime} \circ \partial S K_{j}\right)=\operatorname{deg}_{2}\left(f_{j} \circ \partial S K_{j}\right)$ for $j=1,2,3$. We cannot apply the method of Step 2 of that proof directly because there are three boundary circles instead of two and the circles are not concentric. But we can apply the method in Step 2 as above, separately in the following two parts of $S K H$ :

$$
\begin{aligned}
& S K H_{1}=\{(r, s, \mathbf{x}) \in S K H \mid-9 \leq r \leq 9,0 \leq s \leq 9\} \quad \text { and } \\
& S K H_{2}=\{(r, s, \mathbf{x}) \in S K H \mid-9 \leq r \leq 9, \quad-9 \leq s \leq 0\},
\end{aligned}
$$

choosing the same subset of the intersection $S K H_{1} \cap S K H_{2}$ in the definition of the extension using the product structure. See Figure 2.5. Both of the $S K H_{i}$ are homeomorphic to $S A H$.

(C) Let $B_{3}=\{(0, r, \mathbf{x}) \in S K G \mid-10 \leq r \leq-5\}, B_{1}=\{(0, r, \mathbf{x}) \in$ $S K G \mid-3 \leq r \leq 3\}$ and $B_{2}=\{(0, r, \mathbf{x}) \in S K G \mid 5 \leq r \leq 10\}$. Then each $B_{j}$ is homeomorphic to $D^{m}$, and from the above conventions about the orientations, the restriction of $\psi^{\prime}$ to $\partial B_{j}$ has an extension on $B_{j}$, for $j=2,3$, if and only if $\operatorname{deg}_{1}\left(f_{1} \circ \partial S K_{1}\right)+\operatorname{deg}_{1}\left(f_{j} \circ S K_{j}\right)=$ 0 . See Figure 2.5.

(D) By applying twice the method in Step 2 from the proof of Proposition 2.2, once for $K_{1}, K_{2}$, and the second time for $K_{1}, K_{3}$ we can accomplish the following: $\operatorname{deg}_{2}\left(f_{j}^{\prime} \circ \partial S K_{j}\right)=0$ for $j=2,3$. $\operatorname{deg}_{2}\left(f_{1}^{\prime} \circ \partial S K_{1}\right)=\operatorname{deg}_{2}\left(f_{1} \circ \partial S K_{1}\right)+\operatorname{deg}_{2}\left(f_{2} \circ \partial S K_{2}\right)+\operatorname{deg}_{2}\left(f_{3} \circ \partial S K_{3}\right)$, and $\operatorname{deg}_{1}\left(f_{j}^{\prime} \circ \partial S K_{j}\right)=\operatorname{deg}_{1}\left(f_{j} \circ \partial S K_{j}\right)$ for $j=1,2,3$.

(E) Let $\psi$ have an extension. This implies that $\psi^{\prime}$ has an extension. Then, (C) implies that $\operatorname{deg}_{1}\left(f_{1} \circ \partial S K_{1}\right)+\operatorname{deg}_{1}\left(f_{j} \circ S K_{j}\right)$ $=0$, for $j=2,3$, i.e. (1) is satisfied. Moreover, (B) implies that $\operatorname{deg}_{1}\left(f_{j}^{\prime} \circ \partial S K_{j}\right)=0$ for $j=1,2,3$, and (D) implies that $\operatorname{deg}_{2}\left(f_{j}^{\prime} \circ \partial S K_{j}\right)=0$ for $j=2,3$. This, together with Proposition 1.14, implies that the restrictions of $\varphi^{\prime}$ to $\partial S K_{j}, j=2,3$, have extensions. By $H E T$, these extensions can be chosen to map $\partial S K_{j}, j=2,3$, to $\mathscr{N}(m-1)$. So, we can replace $S K G$ by $S D$ (see (2.3)), i.e. we can extend the map $\varphi^{\prime}$ on the 1-regular neighborhoods 
of $D_{2}$ and $D_{3}$, by mapping them to $\mathscr{N}(m-1)$. Then Proposition 2.1 implies that $\operatorname{deg}_{2}\left(f_{1}^{\prime} \circ \partial S K_{1}\right)=0$. Hence, $\operatorname{deg}_{2}\left(f_{1}^{\prime} \circ \partial S K_{1}\right)=$ $\operatorname{deg}_{2}\left(f_{1} \circ \partial S K_{1}\right)+\operatorname{deg}_{2}\left(f_{2} \circ \partial S K_{2}\right)+\operatorname{deg}_{2}\left(f_{3} \circ \partial S K_{3}\right)=0$, i.e. the condition (2) is satisfied.

(F) Conversely, let the conditions (1) and (2) be satisfied. Then, by (A), (B) and (D), we have $\operatorname{deg}_{i}\left(f_{j}^{\prime} \circ \partial S K_{j}\right)=0$ for the map $\varphi^{\prime}$, $i=1,2, j=1,2,3$. So, Proposition 1.14 implies that each of the maps $f_{j}^{\prime}$ has an extension $\bar{f}_{j}: S K_{j} \rightarrow S^{m-1}$, which together with the restriction of $\varphi^{\prime}$ to $S K H$ give an extension $\xi^{\prime}: S K G \rightarrow S^{m-1}$ for $\psi^{\prime}$. Since $\psi$ and $\psi^{\prime}$ are homotopic, it follows that $\psi$ has an extension $\xi: S K G \rightarrow S^{m-1}$.

III. Indices 1 and 2. Let $F: X \times I \rightarrow X$ be given, where $X \subseteq$ $\mathbb{R}^{m}$ is an $m$-dimensional, compact, connected $P L$ oriented manifold, such that $X \times I \subseteq \mathbb{R}^{m} \times \mathbb{R}=\mathbb{R}^{m+1}$, and let $m \geq 4$. We assume that $X$ is oriented by the induced orientation from $\mathbb{R}^{m}$, and $X \times I$ has the product orientation. Let $S E: S^{1} \times D^{m} \rightarrow \mathbb{R} \times \mathbb{R}^{m}$, be the standard embedding defined by (2.1). For each embedded oriented circle $C \subseteq X \times I$ and a regular neighborhood $W$ of $C$ in $X \times I$ we choose the isotopy class $\{\varphi\}$ of orientation preserving embeddings of pairs, $\varphi:\left(S^{1} \times D^{m}, S^{1} \times\{\boldsymbol{0}\}\right) \rightarrow(W, C)$, by isotoping the standard embedding $S E$. Such a choice exists, because every two oriented embedded circles in $\mathbb{R}^{m+1}$ are isotopic, for $m \geq 3$.

Let $P: X \times I \rightarrow X$ be the projection, defined by $P(x, t)=x$, for every $x \in X$ and $t \in I$. A fixed point of the map $F$ is a point $(x, t) \in$ $X \times I$, such that $F(x, t)=x=P(x, t)$. The set of fixed points of $F$ is denoted by $\operatorname{Fix}(F)$, i.e. $\operatorname{Fix}(F)=\{(x, t) \in X \times I \mid F(x, t)=x\}$. Let $C \subseteq X \times I$ be an isolated circle of fixed points, i.e. a circle of fixed points, which has a small enough neighborhood $W$, such that the only fixed points of $F$ in $W$ are the points of $C$. Since $X \subseteq \mathbb{R}^{m}$, the map $P-F:(W, C) \rightarrow\left(D_{\varepsilon}^{m}, \mathbf{0}\right)$ is defined, and we denote it by $\rho(F)$, where $D_{\varepsilon}^{m}=\left\{\mathbf{x} \in \mathbb{R}^{m}|| \mathbf{x} \mid \leq \varepsilon\right\}$. Since $C$ is an isolated circle of fixed points, we have $\rho(F)^{-1}(\mathbf{0})=C$. There are two orientations on $C$; we denote them by $O_{1}$ and $O_{2}$. Let $\varphi$ be an embedding from the chosen isotopy class for such an oriented circle $C$ and its neighborhood $W$. So we have a map $\zeta \circ \rho(F) \circ \varphi: S^{1} \times D^{m} \rightarrow D^{m}$, where $\zeta: D_{\varepsilon}^{m} \rightarrow D^{m}$ is the homeomorphism defined by multiplication by $1 / \varepsilon$. Let $\mu(F): S^{1} \times S^{m-1} \rightarrow S^{m-1}$ be the map $\mu(F)=\xi \circ \zeta \circ$ $\rho(F) \circ \varphi^{\prime}$ where $\xi: D^{m} \backslash\{\mathbf{0}\} \rightarrow S^{m-1}$ is defined by $\xi(\mathbf{x})=\frac{1}{|\mathbf{x}|} \cdot \mathbf{x}$, and $\varphi^{\prime}$ is the restriction of $\varphi$ to $S^{1} \times S^{m-1}$. 
Proposition 3.1. Let $\mu(F)$ and $\mu^{\prime}(F)$ be defined as above, for the two orientations on $C$. Then, $\operatorname{deg}_{1}(\mu(F))=-\operatorname{deg}_{1}\left(\mu^{\prime}(F)\right)$, and $\operatorname{deg}_{2}(\mu(F))=\operatorname{deg}_{2}\left(\mu^{\prime}(F)\right)$.

Proof. Let $\varphi_{1}$ and $\varphi_{2}$ be two embeddings from the chosen isotopy classes for the oriented circles $\left(C, O_{1}\right)$ and $\left(C, O_{2}\right)$.

Let $\gamma: S^{1} \rightarrow S^{1}$ and $\delta: D^{m} \rightarrow D^{m}$ be orientation reversing homeomorphisms, and let $\psi=(\gamma, \delta)$. Then $\varphi_{2}$ and $\varphi_{1} \circ \psi$ are in the same isotopy class, and moreover they are homotopic.

In the definition of $\mathrm{deg}_{1}$ we need only the orientation of $S^{m-1}$, not of $S^{1}$. So, since $\mu(F)$ and $\mu^{\prime}(F)$ differ only in $\varphi_{1}$ and $\varphi_{2}$, we need only to examine the restrictions $\alpha_{1}$ of $\varphi_{1}$ and $\alpha_{2}$ of $\varphi_{2}$ to $\mathscr{N}(1) \times D^{m}$. Because $\varphi_{2}$ and $\varphi_{1} \circ \psi$ are isotopic, it follows that $\operatorname{deg}_{1}(\mu(F))=\operatorname{deg}\left(\alpha_{2}\right)=\operatorname{deg}\left(\alpha_{1} \circ \delta\right)=\operatorname{deg}\left(\alpha_{1}\right) \cdot \operatorname{deg}(\delta)=\operatorname{deg}\left(\alpha_{1}\right) \cdot$ $(-1)=-\operatorname{deg}\left(\alpha_{1}\right)=-\operatorname{deg}_{1}\left(\mu^{\prime}(F)\right)$.

Since $\mu(F)$ and $\mu^{\prime}(F)$ differ only in $\varphi_{1}$ and $\varphi_{2}$, and the difference is a homeomorphism of $S^{1} \times S^{m-1}$ which is a product of two homeomorphisms, it follows that $\operatorname{deg}_{2}(\mu(F))=0$ if and only if $\operatorname{deg}_{2}\left(\mu^{\prime}(F)\right)=0$. Hence, $\operatorname{deg}_{2}(\mu(F))=\operatorname{deg}_{2}\left(\mu^{\prime}(F)\right)$.

Definition 3.1. For a chosen orientation $O$ on $C, \operatorname{deg}_{1}(\mu(F))$ will be denoted by $i_{1}(F, C, O)$. We say that an orientation on $C$ is the natural orientation on $C$, if $\operatorname{deg}_{1}(\mu(F)) \geq 0$, and we say that $\operatorname{deg}_{1}(\mu(F))$ for this orientation is index 1 of $F$ at $C$, denoted by $i_{1}(F, C)$.

REMARK 3.2. By the definition, $i_{1}(F, C) \geq 0$. In the case $\operatorname{deg}_{1}(\mu(F))=0$, both of the two orientations on $C$ are natural, or using different words, $C$ does not have a natural orientation.

The following notion is well defined by Proposition 3.1 .

Definition 3.2. We define index 2 of $F$ at $C$, denoted by $i_{2}(F, C)$, to be $\operatorname{deg}_{2}(\mu(F))$.

Proposition 3.3. Let $X, F, C$ and $W$ be as above. Then, $i_{1}(F, C)$ $=0=i_{2}(F, C)$, if and only if $F$ is homotopic to a map $G: X \times I \rightarrow$ $X \operatorname{rel} X \times I \backslash W$, with $G(x, t) \neq x$ for each $(x, t) \in W$.

Proof. Let $i_{1}(F, C)=0=i_{2}(F, C)$. Then $\operatorname{deg}_{1}(\mu(F))=0=$ $\operatorname{deg}_{2}(\mu(F))$, and so $\mu(F)$ is homotopic to a map $\kappa: S^{1} \times S^{m-1} \rightarrow$ $S^{m-1}$, defined by $\kappa(\mathbf{z}, \mathbf{x})=f(\mathbf{x})$, where $f: S^{m-1} \rightarrow S^{m-1}$ is a map of degree 0 . The map $f$ has an extension to a map $\bar{f}: D^{m} \rightarrow S^{m-1}$, 
which gives an extension of $\kappa$ to a map $\bar{\kappa}: S^{1} \times D^{m} \rightarrow S^{m-1}$. This gives a homotopy $H: W \times I \rightarrow D_{\varepsilon}^{m}$ rel $\partial W$, from $\rho(F)$ to a map $\bar{\rho}: W \rightarrow D_{\varepsilon}^{m} \backslash\{\boldsymbol{0}\}$. So, for $G=P-\bar{\rho}$, we have that $F$ is homotopic to $G \operatorname{rel} X \times I \backslash W$, with $G(x, t) \neq x$ for each $(x, t) \in W$. Conversely, let $F$ be homotopic to a map $G \operatorname{rel} X \times I \backslash W$, such that $G(x, t) \neq x$ for each $(x, t) \in W$. Let $\rho(G)=P-G$, and let $\mu(G)=\xi \circ \zeta \circ \rho(G) \circ \varphi$. Then $\mu(F)=\mu(G)$, and since $\rho(G)$ has an extension from $W$ to $D_{\varepsilon}^{m}$, it follows that $\mu(F): S^{1} \times S^{m-1} \rightarrow S^{m-1}$ has an extension from $S^{1} \times D^{m}$ to $S^{m-1}$. This implies that $\operatorname{deg}_{1}(\mu(F))=0=$ $\operatorname{deg}_{2}(\mu(F))$.

Definition 3.3. Let $A$ be an isolated fixed point of $F: X \times I \rightarrow$ $X$. Let $V$ be a small $(m+1)$-ball neighborhood of $A$ in $X \times I$, such that $F(x) \neq P(x)$ for every $x \in V \backslash\{A\}$. Let $\varphi: D^{m+1} \rightarrow$ $V$ be a homeomorphism, and let $\mu(F): S^{m} \rightarrow S^{m-1}$ be the map $\xi \circ \zeta \circ(P-f) \circ \varphi^{\prime}$, where $\varphi^{\prime}$ is the restriction of $\varphi$ to $S^{m}=\partial D^{m+1}$. Define index 2 of $F$ at $A$, denoted by $i_{2}(F, A)$ to be the element $[\mu(F)] \in \pi_{m}\left(S^{m-1}\right)$.

Let $C$ be an isolated circle of fixed points of $F: X \times I \rightarrow X, B$ be an embedded disk in $\operatorname{int}(X \times I)$, with $\partial B=C$, and $H:(X \times I \times$ $\{0\}) \cup(B \times I) \rightarrow X$ be a partial homotopy such that $H(x, 0)=F(x)$, for all $x \in X \times I, H(x, 1)=x$ for all $x \in B$, and $H(x, t)=x$ for all $x \in C$ and all $t \in I$.

Proposition 3.4. Let $F, C, B$ and $H$ be as above. Then there is a neighborhood $N$ of $B$, and a map $G^{\prime}: X \times I \rightarrow X$, homotopic to $F \operatorname{rel} X \times I \backslash N$, such that $\operatorname{Fix}\left(G^{\prime}\right)=(\operatorname{Fix}(F) \backslash C) \cup\{A\}$, and $i_{2}(F, C)=$ $i_{2}\left(G^{\prime}, A\right)$, where $A$ is an isolated fixed point of $G^{\prime}$. Moreover, if $i_{2}(F, C)=0$, there is a neighborhood $N$ of $B$, and a map $G: X \times I \rightarrow$ $X$, homotopic to $F \operatorname{rel} X \times I \backslash N$, such that $\operatorname{Fix}(G)=\operatorname{Fix}(F) \backslash C$.

Proof. Let $N$ be a regular neighborhood of $B$ in $X \times I$, such that $\operatorname{Fix}(F) \cap N=C$. Let $\varepsilon>0$ be such that the $2 \varepsilon$-neighborhood $N_{2 \varepsilon}(B)$ is contained in int $N$, and the $2 \varepsilon$-neighborhood $N_{2 \varepsilon}(X)$ of $X$ in $\mathbb{R}^{m}$ retracts to $X$. Then, by the same methods as in [DG], $F$ is homotopic to a map $F^{\prime}$ rel $X \times I \backslash N$, and there exists a regular neighborhood $N^{\prime}$ of $B$, such that $N^{\prime} \subseteq N_{\varepsilon}(B)$, and $F^{\prime}$ and $P$ are $\varepsilon$-close on $N^{\prime}$, i.e. for each $x \in N^{\prime}, d\left(F^{\prime}(x), P(x)\right)<\varepsilon$. So we have a map $\left(P-F^{\prime}\right) \mid: N^{\prime} \backslash C \rightarrow N_{\varepsilon}(\mathbf{0}) \backslash\{\boldsymbol{0}\} \subseteq \mathbb{R}^{m} \backslash\{\mathbf{0}\}$. Since $N^{\prime}$ is homeomorphic to $D^{m+1}$, we can choose a point $A \in \operatorname{int} N^{\prime}$, such that the map $\left(P-F^{\prime}\right)$, restricted to $\partial N^{\prime}$ has an extension $K^{\prime}: N^{\prime} \rightarrow N_{\varepsilon}(\mathbf{0})$, 
with $\left(K^{\prime}\right)^{-1}(\mathbf{0})=A$. Now, let $G^{\prime}: X \times I \rightarrow \mathbb{R}^{n}$ be defined by: $G^{\prime}=F^{\prime}$ outside $N^{\prime}$, and $G^{\prime}=P-K^{\prime}$ on $N^{\prime}$. Then $F$ is homotopic to $G^{\prime} \operatorname{rel} X \times I \backslash N$, with $\operatorname{Fix}\left(G^{\prime}\right)=(\operatorname{Fix} F \backslash C) \cup\{A\}$. Because $F$ is homotopic to $F^{\prime}$ by a special homotopy [DG], it follows that $F^{\prime}$ has $C$ as an isolated circle of fixed points, and $i_{2}\left(F^{\prime}, C\right)=i_{2}(F, C)$. Next: because both the standard model $S D$ and $N^{\prime}$ are $(m+1)$-balls, the isotopy of the standard embedding $S E$ for $W$ can be extended to an isotopy which sends $S D$ to $N^{\prime}$, which together with Proposition 2.1 implies that $i_{2}\left(F^{\prime}, C\right)=0$ if and only if $\left(P-F^{\prime}\right)$, restricted to $\partial N^{\prime}$, has an extension $K: N^{\prime} \rightarrow N_{\varepsilon}(\mathbf{0}) \backslash\{\mathbf{0}\}$, i.e. if and only if $i_{2}\left(G^{\prime}, A\right)=0$. Hence, $i_{2}(F, C)=i_{2}\left(G^{\prime}, A\right)$.

If $i_{2}(F, C)=0$, then $i_{2}\left(G^{\prime}, A\right)=0$, and so, the restriction of $\left(P-G^{\prime}\right)$ to $\partial N^{\prime}$ has an extension $K: N^{\prime} \rightarrow N_{\varepsilon}(\mathbf{0}) \backslash\{\mathbf{0}\}$. Let $G$ be defined using $K$, in the same way as $G^{\prime}$ was defined using $K^{\prime}$. Then $F$ is homotopic to $G \operatorname{rel} X \times I \backslash N$ and $\operatorname{Fix}(G)=\operatorname{Fix}(F) \backslash C$.

Next, let $C \subseteq X \times I$ be an embedded circle on which $F$ and $P$ are $\varepsilon$-close, as mentioned above, i.e. $N_{2 \varepsilon}(X)$ retracts to $X$ in $\mathbb{R}^{m}$, and $d(F(x), P(x)) \leq \varepsilon$ for each $x \in W$ where $W$ is a small regular neighborhood of $C$ in $X \times I$. Assume that for each $x \in \partial W, F(x) \neq$ $P(x)$. Choose any orientation $O$ for $C$, and let $\varphi$ be an embedding from the chosen isotopy class of embeddings for $(W, C)$. Let $f=$ $P-F$, let $g$ denote the restriction of $f \circ \varphi$ to $S^{1} \times S^{m-1}$, and let $\operatorname{deg}_{1}(g)=k, k \in \mathbb{Z}$.

Theorem 3.5. Let $X, F, C, W, \varphi, f, g, k$ be as above, let $m \geq$ 4 , and for $r \geq 1$, let $t_{j}, j=1, \ldots, r$, be arbitrary numbers with $\sum t_{i}=\operatorname{deg}_{1}(g)$. Then $F$ is homotopic to a map $G: X \times I \rightarrow X$, $\operatorname{rel} X \times I \backslash W$ such that:

(1) $G$ has $r$ isolated circles of fixed points $C_{1}, C_{2}, \ldots, C_{r}$ in $W$, all of them "parallel" to $C$;

(2) $G$ does not have other fixed points in $W$;

(3) $\sum i_{2}\left(G, C_{j}\right)=\operatorname{deg}_{2}(g)$, where the sum is in $\pi_{m}\left(S^{m-1}\right)$;

(4) If we orient $C_{j}$ with an orientation $O_{j}$ compatible with the orientation $O$ on $C$ (meaning that $(C, O)$ and $\left(C_{j}, O_{j}\right)$ determine the same element in the first homology group $\left.H_{1}(W)\right)$ then, for each $j=1,2, \ldots, r, i_{1}\left(G, C_{j}, O_{j}\right)=t_{j}$; and

(5) If for each $j, t_{j}=1$, then it is possible to make the circles $C_{j}$ transverse, which means that the graph of $G$ and the graph of the projection $P: X \times I \rightarrow X$, intersect transversely in $X \times I \times X$ at each $C_{j}$. 
Proof. Let $V=\varphi\left((0,1) \times D^{m}\right) \subseteq W$, and let $W^{\prime}=\varphi\left(S^{1} \times B^{m}\right)$, where $B^{m}=D_{1 / 2}^{m}$. Then, by the creating procedure of fixed points [BJ], there is a map $\xi: B^{m} \rightarrow B^{m}$, with $r$ fixed points, $L_{1}, \ldots, L_{r}$, whose indices are $t_{1}, \ldots, t_{r}$ respectively, with their sum equal to $k$. Moreover, if for each $j, t_{j}=1$, then the fixed points $L_{j}$ are transverse. Let $h=\mathrm{id}-\xi: B^{m} \rightarrow D_{\varepsilon}^{m}$, and let $h \mid$ be its restriction to $\partial\left(B^{m}\right)$. Then $\operatorname{deg}(h \mid)=k$ with $B^{m}$ and $D_{\varepsilon}^{m}$ oriented by the induced orientation from $D^{m}$. Let $B_{1}, \ldots, B_{r}$ be disjoint ball neighborhoods of $L_{1}, \ldots, L_{r}$ in $B^{m}$. Let $C_{j}=\varphi\left(S^{1} \times L_{j}\right)$, and let $V_{j}=\varphi\left(S^{1} \times B_{j}\right)$. Using the map $h$, we define a map $\psi: S^{1} \times B^{m} \rightarrow D_{\varepsilon}^{m}$, by $\psi(z, x)=$ $h(x)$. By the definition of $\psi$, we have that $\operatorname{deg}_{1}(\psi \mid)=k=\operatorname{deg}_{1}(g)$, and $\operatorname{deg}_{2}(\psi \mid)=0$, where $\psi \mid$ is the restriction of $\psi$ to $S^{1} \times \partial B^{m}$. We will consider two cases: when $\operatorname{deg}_{2}(g)=0$, and when $\operatorname{deg}_{2}(g) \neq 0$.

Case 1. If $\operatorname{deg}_{2}(g)=0$, then $\psi \mid$ is homotopic to $g$, which shows that there is a map $\psi^{\prime}: S^{1} \times D^{m} \rightarrow D_{\varepsilon}^{m}$, extending $\psi$ and $g$, and such that $\left(\psi^{\prime}\right)^{-1}(\mathbf{0})=S^{1} \times\left(L_{1} \cup \cdots \cup L_{r}\right)$. Then the map $G$ defined by $G=F$ on $X \times I \backslash W$ and by $G=P-\psi^{\prime} \circ \varphi^{-1}$ on $W$, satisfies the conclusions.

Case 2. Let $\operatorname{deg}_{2}(g) \neq 0$. We use a method similar to the one in Step 2 of the proof of Proposition 2.2. First we choose two points $A \in S^{1} \times \partial B^{m}$, and $A^{\prime} \in S^{1} \times \partial B_{1}$, and an arc $\alpha$ from $A$ to $A^{\prime}$ in $S^{1} \times B^{m}$, missing int $B_{1}$ and all the other $B_{j}$ 's. Let $(N(\alpha), N(A), N(B))$ be a regular neighborhood triple of $\left(\alpha, A, A^{\prime}\right)$ in $\left(S^{1} \times B^{m}, S^{1} \times \partial B^{m}, S^{1} \times \partial B_{1}\right)$. We can homotope the map $\psi$ to a map $\lambda$, such that $\lambda(N(\alpha))$ is a point in $D_{\varepsilon}^{m} \backslash\{0\}$. Next we can replace $\lambda$ by another map $\lambda^{\prime}$, defined by $\lambda^{\prime}=\lambda$ outside $N(\alpha) \cup S^{1} \times B_{1}$; the restrictions of $\lambda^{\prime}$ to $N(A)$ and $N\left(A^{\prime}\right)$ give nontrivial elements of $\pi_{m}\left(S^{m-1}\right) ; \lambda^{\prime}$ on $N(\alpha)$ is an extension of the map defined on $\partial N(\alpha)$, and $\lambda^{\prime}$ on $S^{1} \times B_{1}$ is defined by coning the map from $S^{1} \times \partial B_{1}$ to $S^{1} \times \mathbf{O}$, where $\mathbf{O}$ is the center of $B_{1}$. In the case $t_{j}=1$ for each $j$, we redefine $\lambda^{\prime}$ on $S^{1} \times B_{1}$ as follows. Let $\rho$ be the restriction of $\lambda^{\prime}$ to $S^{1} \times \partial B_{1}$. Then $\operatorname{deg}_{1}(\rho)=1$ in $\mathbb{Z}$ and $\operatorname{deg}_{2}(\rho)=1$ in $\mathbb{Z}_{2}$, which implies that $\rho$ is homotopic to the map $h(m-1)$ defined by (1.9). Let $B_{1}^{\prime} \subseteq B_{1}$ be a smaller concentric ball in $B_{1}$ with the same center $\mathbf{O}$. We define $\lambda^{\prime}$ on the closure of $S^{1} \times\left(B_{1} \backslash B_{1}^{\prime}\right)$ via the homotopy from $\rho$ to $h(m-1)$, and on $S^{1} \times B_{1}^{\prime}$ by coning $h(m-1)$ to $S^{1} \times \mathbf{O}$. For $\lambda^{\prime}$ defined in this manner, $\operatorname{deg}_{2}\left(\lambda^{\prime} \mid\right) \neq 0$, where $\lambda^{\prime} \mid$ is the restriction of $\lambda^{\prime}$ to $S^{1} \times \partial B^{m}$, which shows that $\lambda^{\prime} \mid$ is homotopic to $g$. Hence, there is a map $\psi^{\prime}: S^{1} \times D^{m} \rightarrow D_{\varepsilon}^{m}$, extending $\lambda^{\prime}$ 
and $g$, such that $\left(\psi^{\prime}\right)^{-1}(\mathbf{0})=S^{1} \times\left(L_{1} \cup \cdots \cup L_{r}\right)$. Then the map $G$ defined by $G=F$ on $X \times I \backslash W$ and by $G=P-\psi^{\prime} \circ \varphi^{-1}$ on $W$, satisfies the conclusions (1), (2), (3) and (4), because $i_{2}\left(G, C_{j}\right)=0$ for all $j \neq 1$, and $i_{2}\left(G, C_{1}\right)=\operatorname{deg}_{2}(g)$. The map $G$ satisfies conclusion (5) for each circle $C_{j}, j \neq 1$, because the map $\psi$ is defined by $\psi(z, x)=h(x)$. The circle $C_{1}$ is transverse, because for each arc $[A, B] \subseteq S^{1}$, the restriction of the map $h(m-1)$ to $[A, B] \times B_{1}$ is homotopic to the map $\gamma$, defined by $\gamma(z, x)=h(m-1)(A, x)$, and because $\lambda^{\prime}$ was defined on $S^{1} \times B_{1}^{\prime}$ by coning $h(m-1)$ to $S^{1} \times \mathbf{O}$.

Proposition 3.6. Let $X, F, C, W, \varphi, f, g$, and $k$ be as above. Then:

(1) $F$ is homotopic to a map $G_{1}: X \times I \rightarrow X$, rel $X \times I \backslash W$ such that: $C$ is an isolated circle of fixed points for $G_{1}$; and $G_{1}$ does not have other fixed points in $W ; i_{1}\left(G_{1}, C\right)=|k|$; and $i_{2}\left(G_{1}, C\right)=$ $\operatorname{deg}_{2}(g)$.

(2) If $k \neq 0$, then $F$ is homotopic to a map $G_{2}: X \times I \rightarrow X$, rel $X \times I \backslash W$ such that: $G_{2}$ has $|k|$ isolated circles of fixed points $C_{1}, \ldots, C_{|k|}$ in $W$, all of them "parallel" to $C ; G_{2}$ does not have other fixed points in $W ; i_{1}\left(G_{2}, C_{j}\right)=1$ for each $j \in\{1, \ldots,|k|\}$; and $\sum i_{2}\left(G_{2}, C_{j}\right)=\operatorname{deg}_{2}(g)$, where the sum is in $\pi_{m}\left(S^{m-1}\right)$. Moreover, it is possible to make the circles $C_{j}$ transverse.

(3) If $k=0$ and $\operatorname{deg}_{2}(g)=0$, then $F$ is homotopic to a map $G_{3}: X \times I \rightarrow X$, rel $X \times I \backslash W$ such that $G_{3}$ has no fixed points in $W$.

(4) If $k=0$, and $\operatorname{deg}_{2}(g) \neq 0$, then $F$ is homotopic to a map $G_{4}: X \times I \rightarrow X$, rel $X \times I \backslash W$ such that $G_{4}$ has only one isolated fixed point $A$ in $W$, and $i_{2}\left(G_{4}, A\right)=\operatorname{deg}_{2}(g)$.

Proof. (1) and (2) follow directly from Theroem 3.5.

(3) follows from Theorem 3.5 and Proposition 3.3.

(4) follows from Theorem 3.5 and Proposition 3.4.

There is a converse to Proposition 3.4.

Proposition 3.7. Let $A$ be an isolated fixed point of $F$. Then there is a regular neighborhood $N$ of $A$, and a map $G: X \times I \rightarrow X$, homotopic to $F \operatorname{rel} X \times I \backslash N$, such that $\operatorname{Fix}(G)=(\operatorname{Fix} F \backslash\{A\}) \cup C$, and $i_{2}(G, C)=i_{2}(F, A)$, where $C$ is an isolated circle of fixed points of $G$ which bounds a disk $B$ in $N$, on which $G$ and $P$ are $\varepsilon$-close. 
Moreover, $i_{1}(G, C)$ can be chosen to be any number $\geq 0$, and if $i_{1}(G, C)=1$, then the circle $C$ can be made transverse.

Proof. Let $N$ be a small regular neighborhood of $A$, such that $F$ does not have other fixed points in $N$, and $F$ and $P$ are $\varepsilon$-close on $N$. Let $C$ be a circle in $N$ missing $A$, and let $W$ be a regular neighborhood of $C$ in $N$. Then Theorem 3.5 implies that $F$ is homotopic to a map $H$ rel $X \times I \backslash W$, such that: $H$ has two more circles of fixed points $C$ and $C^{\prime} ; i_{2}\left(H, C^{\prime}\right)=i_{2}(H, C)=i_{2}(F, A) ; i_{1}(H, C)$ is a previously chosen number $\geq 0$; and $C^{\prime}$ bounds a disk $B$ missing $A$ and $C$, on which $H$ and $P$ are $\varepsilon$-close; and in the case $i_{1}(H, C)=1$, the circle $C$ is transverse. Then, Proposition 3.4 implies that $H$ is homotopic to a map $H^{\prime}$ rel $X \times I \backslash N^{\prime}$ where $N^{\prime}$ is a small regular neighborhood of $B$, such that $\operatorname{Fix}\left(H^{\prime}\right)=\left(\operatorname{Fix}(H) \backslash C^{\prime}\right) \cup\left\{A^{\prime}\right\}$ where $A^{\prime}$ is an isolated fixed point of $H^{\prime}$ in $N$ with $i_{2}\left(H^{\prime}, A^{\prime}\right)=$ $i_{2}\left(H^{\prime}, A\right)$. Then $H^{\prime}$ is homotopic to a map $G \operatorname{rel} X \times I \backslash N^{\prime \prime}$, where $N^{\prime \prime}$ is a small regular neighborhood of an arc from $A$ to $A^{\prime}$ in $N$, missing $C$, such that $\operatorname{Fix}(G)=\operatorname{Fix}\left(H^{\prime}\right) \backslash\left\{A, A^{\prime}\right\}$, i.e. $\operatorname{Fix}(G)=$ $(\operatorname{Fix}(F) \backslash\{A\}) \cup C$.

Proposition 3.8. Let $f: K G \rightarrow X \times I$ be an embedding, and let id: $K G \rightarrow X \times I$ be the identity embedding of $K G$ in $\mathbb{R}^{m+1}, m \geq 4$, where $K G$ is defined by (2.9). Then, $f(K G)$ is isotopic to $K G$.

Proof. Since $K G$ has a one dimensional core, and $m \geq 4$, it follows that $f$ and id are homotopic embeddings. This homotopy implies the isotopy for $m \geq 5$. For $m=4, f(K G)$ can be isotoped to a new position denoted again by $f(K G)$, such that $f(K G) \cap K G=\varnothing$. The (exterior) circles $K_{1}$ and $f\left(K_{1}\right)$ are homotopic, and the homotopy can be homotoped to an embedding $h\left(S^{1} \times I\right)$. Since $K_{2}, K_{3}, f\left(K_{2}\right)$ and $f\left(K_{3}\right)$ are nullhomotopic in $\mathbb{R}^{5}$, they bound disjoint embedded disks, whose interiors are disjoint from $h\left(S^{1} \times I\right)$. These disks, together with $h\left(S^{1} \times I\right)$, form an embedded $S^{2}$ in $\mathbb{R}^{5}$, which is unknotted, ([RS]), and bound a standard $D^{3}$ in $\mathbb{R}^{5}$. Since the circles $K_{2}, K_{3}, f\left(K_{2}\right)$ and $f\left(K_{3}\right)$ are standard in $S^{2}$, we obtain a standard $K G \times I$ in $\mathbb{R}^{5}$, such that $K G \times\{0\}=K G$ and $K G \times\{1\}$ is isotopic to $f(K G)$. Hence $K G$ is isotopic to $f(K G)$.

Let $X, F$ be as above, let $C_{2}, C_{3}$ be two circles of fixed points of $F$. Let $f(K G)$ be an embedded disk with two holes in $X \times I$, such that $C_{j}=f\left(K_{j}\right), j=2,3$, and $f(K G)$ has a regular neighborhood in $X \times I$ whose intersection with $\operatorname{Fix}(F)$ is $C_{2} \cup C_{3}$. Let $C_{1}=f\left(K_{1}\right)$, 
and let $H:(X \times I \times\{0\}) \cup(f(K G) \times I) \rightarrow X$ be a partial homotopy such that $H(x, 0)=F(x)$, for all $x \in X \times I, H(x, 1)=x$ for all $x \in f(K G)$, and $H(x, t)=x$ for all $x \in C_{j}, j=2,3$ and all $t \in I$.

Proposition 3.9. Let $F, C_{j}, f(K G)$ and $H$ be as above. Let the natural orientation of $C_{j}$ agree with the induced orientation from $f(K G)$, and let $i_{1}\left(F, C_{2}\right)=i_{1}\left(F, C_{3}\right)$. Then there is a neighborhood $N$ of $f(K G)$, and a map $G: X \times I \rightarrow X$, homotopic to $F \operatorname{rel} X \times I \backslash N$, such that: $C_{1}$ is an isolated circle of fixed points of $G$ whose natural orientation agrees with the one induced from $f(K G) ; \operatorname{Fix}(G)=$ $\left(\operatorname{Fix}(F) \backslash\left(C_{2} \cup C_{3}\right)\right) \cup C_{1} ; \quad i_{1}\left(G, C_{1}\right)=i_{1}\left(F, C_{2}\right) ;$ and $i_{2}\left(G, C_{1}\right)=$ $i_{2}\left(F, C_{2}\right)+i_{2}\left(F, C_{3}\right)$. Moreover, if $C_{2}$ and $C_{3}$ are transverse, then $C_{1}$ can be made transverse.

Proof. Let $N$ be a regular neighborhood of $f(K G)$ in $X \times I$, such that $\operatorname{Fix}(F) \cap N=C_{2} \cup C_{3}$. Let $\varepsilon>0$ be such that the $2 \varepsilon$-neighborhood $N_{2 \varepsilon}(f(K G))$ is contained in int $N$, and the $2 \varepsilon$ neighborhood $N_{2 \varepsilon}(X)$ of $X$ in $\mathbb{R}^{m}$ retracts to $X$. Then, by the same methods as in [DG], $F$ is homotopic to a map $F^{\prime} \operatorname{rel} X \times I \backslash N$, and there exists a regular neighborhood $N^{\prime}$ of $f(K G)$, such that $N^{\prime} \subseteq N_{\varepsilon}(f(K G))$, and $F^{\prime}$ and $P$ are $\varepsilon$-close on $N^{\prime}$, i.e. for each $x \in N^{\prime}, d\left(F^{\prime}(x), P(x)\right)<\varepsilon$. Let $K_{1}^{\prime}=\{(\mathbf{z}, \mathbf{0}) \in K G|| \mathbf{z} \mid=8\}$, be a circle in $K G$ parallel to $K_{1}$, and let $C_{1}^{\prime}=f\left(K_{1}^{\prime}\right)$. Let $W$ be a small regular neighborhood of $C_{1}$ containing $C_{1}^{\prime}$, contained in $N^{\prime}$, and missing $C_{2}, C_{3}$. Then, by Theorem 3.5, $F^{\prime}$ is homotopic to a map $G^{\prime} \operatorname{rel} X \times I \backslash W$, such that: $\operatorname{Fix}\left(G^{\prime}\right)=\operatorname{Fix}(F) \cup C_{1} \cup C_{1}^{\prime}$; $i_{1}\left(G^{\prime}, C_{1}\right)=i_{1}\left(F, C_{1}^{\prime}\right)=i_{1}\left(F, C_{2}\right)$; the natural orientations on $C_{1}$, $C_{1}^{\prime}$ are opposite, i.e. they determine opposite elements in $H_{1}(W)$; $i_{2}\left(G^{\prime}, C_{1}\right)=i_{2}\left(G^{\prime}, C_{1}^{\prime}\right)=i_{2}\left(F, C_{2}\right)+i_{2}\left(F, C_{3}\right)$; the natural orientation of $C_{1}$ agrees with the one induced from $f(K G)$, i.e. $f\left(K_{1}\right)$; and if $C_{2}, C_{3}$ are transverse, then $C_{1}$ and $C_{1}^{\prime}$ can be made transverse. Let $f^{\prime}: K G \rightarrow X \times I$ be the embedding obtained from $f$ by pushing in the collar from $K_{1}$ to $K_{1}^{\prime}$. Let $N^{\prime \prime}$ be a regular neighborhood of $f^{\prime}(K G)$ contained in $N^{\prime}$. Let $\lambda: S K G \rightarrow N^{\prime}$ be a homeomorphism obtained from the isotopy between $K G$ and $f^{\prime}(K G)$, and let $\lambda^{\prime}$ be its restriction to $\partial S K \cup S K H$ (see II). So we have a map $\left(P-G^{\prime}\right) \circ \lambda: \partial S K \cup S K H \rightarrow D_{\varepsilon}^{m} \backslash\{\mathbf{0}\}$, i.e. a map $\xi \circ \zeta \circ\left(P-G^{\prime}\right) \circ$ $\lambda^{\prime}: \partial S K \cup S K G \rightarrow S^{m-1}$, where $\zeta: D_{\varepsilon}^{m} \rightarrow D^{m}$ is the homeomorphism defined by multiplication by $1 / \varepsilon$, and $\xi: D^{m} \backslash\{\boldsymbol{0}\} \rightarrow S^{m-1}$ is defined by $\xi(\mathbf{x})=\frac{1}{|\mathbf{x}|} \cdot \mathbf{x}$. From the choices in the creation of the new circles of fixed points $C_{1}, C_{1}^{\prime}$, it follows that for the map $\varphi=\xi \circ \zeta \circ\left(P-G^{\prime}\right) \circ \lambda^{\prime}$ 
the restrictions $f_{j}$ to $\partial S K_{j}$ and $\psi$ to $\partial S K G$, satisfy the conditions (1) and (2) of Proposition 2.4. This implies that the restriction of the map $\left(P-G^{\prime}\right)$ to $\partial N^{\prime \prime}$ has an extension $\varphi^{\prime}: N^{\prime} \rightarrow D_{\varepsilon}^{m} \backslash\{\boldsymbol{0}\}$, and the map $G: X \times I \rightarrow X$ defined by: $G=G^{\prime}$ on $X \times I \backslash N^{\prime \prime}$ and $G=P-\varphi^{\prime}$ on $N^{\prime \prime}$, satisfies the conclusion of the proposition.

In the preceding proposition, we replaced two circles of fixed points by one. A similar argument holds for replacing one circle of fixed points by two.

Let $X, F$ be as above, let $C_{1}$ be an isolated circle of fixed points of $F$. Let $f(K G)$ be an embedded disk with two holes in $X \times I$, such that $C_{1}=f\left(K_{1}\right)$, and $f(K G)$ has a regular neighborhood in $X \times I$ whose intersection with $\operatorname{Fix}(F)$ is $C_{1}$. Let $C_{j}=f\left(K_{j}\right), j=2,3$, and let $H:(X \times I \times\{0\}) \cup(f(K G) \times I) \rightarrow X$ be a partial homotopy such that $H(x, 0)=F(x)$, for all $x \in X \times I, H(x, 1)=x$ for all $x \in f(K G)$, and $H(x, t)=x$ for all $x \in C_{1}$ and all $t \in I$.

Proposition 3.10. Let $F, C_{j}, f(K G)$ and $H$ be as above, let the natural orientation of $C_{1}$ and the induced orientation from $f(K G)$, i.e. from $f\left(K_{j}\right)$ agree. Then there is a neighborhood $N$ of $f(K G)$, and a map $G: X \times I \rightarrow X$, homotopic to $F \operatorname{rel} X \times I \backslash N$, such that: $C_{2}, C_{3}$ are circles of fixed points of $G$ whose natural orientation agrees with the one induced from $f(K G) ; \operatorname{Fix}(G)=\left(\operatorname{Fix}(F) \backslash C_{1}\right) \cup C_{2} \cup C_{3}$; $i_{1}\left(G, C_{2}\right)=i_{1}\left(G, C_{3}\right)=i_{1}\left(F, C_{1}\right)$; and $i_{2}\left(G, C_{2}\right)+i_{2}\left(G, C_{3}\right)=$ $i_{2}\left(F, C_{1}\right)$. Moreover, if $C_{1}$ is transverse, then $C_{2}, C_{3}$ can be made transverse.

Proof. The proof is the same as the proof of Proposition 3.9 except that we have to use Theorem 3.5 twice, once to create two circles of fixed points $C_{2}, C_{2}^{\prime}$, and once more to create the other two circles of fixed points $C_{3}, C_{3}^{\prime}$.

IV. One parameter Nielsen fixed point theory. Let $F: X \times I \rightarrow X$, $X \subseteq \mathbb{R}^{n}$ an $m$-dimensional, compact connected, oriented manifold, $m \geq 4$ be as in III. Let $A, B$ be two fixed points of $F$. It is said that $A$ and $B$ are in the same fixed point class if there is an arc $\alpha:[0,1] \rightarrow X \times I$ from $A$ to $B$ and a homotopy between $F \circ \alpha$ and $P \circ \alpha$ rel $\{0,1\}$. The relation of being in the same fixed point class is an equivalence. It follows directly from the definition, that if $A, B$ belong to a circle of fixed points then they are in the same fixed point class. Two circles of fixed points are in the same fixed point class if and only if their points are in the same fixed point class. 
Next we recall some definitions and facts, and improve Theorems 9.8 and 10.1 from [DG], where it was assumed tht $F$ is transverse to $P$, which implies that for each isolated circle of fixed points $C$, $i_{1}(F, C)=1$.

Let $E$ be the space of all (continuous, not necessarily PL) paths $\omega(t)$ in $X \times I \times X$ from the graph $\Gamma(F)=\{(x, t, F(x, t)) \mid(x, t) \in$ $X \times I\}$ of $F$ to the graph $\Gamma(P)=\{(x, t, x) \mid(x, t) \in X \times I\}$ of $P$, i.e. maps $\omega:[0,1] \rightarrow X \times I \times X$, such that $\omega(0) \in \Gamma(F)$ and $\omega(1) \in$ $\Gamma(P)$. Let $C_{1}, \ldots, C_{k}$ be isolated circles in $\operatorname{Fix}(F) \cap \operatorname{int}(X \times I)$, oriented by the natural orientations, and let $V=\bigcup C_{j}$. Then $V$ determines a family of circles $V^{\prime}$ in $E$ via the constant paths in $E$, i.e. each oriented isolated circle of fixed points $C: S^{1} \rightarrow X \times I$ of $F$ determines an oriented circle $C^{\prime}: S^{1} \rightarrow E$ defined by $C^{\prime}(\mathbf{z})=$ $\operatorname{con}(C(\mathbf{z}))$ where $\operatorname{con}(C(\mathbf{z}))$ is the constant path at $C(\mathbf{z})=\left(x, t_{0}\right)$, i.e. $\operatorname{con}(C(\mathbf{z}))(t)=\left(x, t_{0}, x\right)$ for each $t \in[0,1]$. The definitions of a fixed point class and $E$ imply that two fixed points $A$ and $B$ are in a single fixed point class if and only if the correspondent points $A^{\prime}$ and $B^{\prime}$ in $E$ are in a single path component of $E$ ([DG]). Since any two points $A, B$ from a circle of fixed points are in a single fixed point class, it follows that a family $V$ of circles of fixed points is in a single fixed point class if and only if there is a compact orientable surface $S_{0}$ and a map $\vartheta: S_{0} \rightarrow E$, such that a part of $\partial S_{0}$ is mapped homeomorphically to $V^{\prime}$. As it is shown in [DG], such a surface $S_{0}$ and a map $\vartheta$ exist if and only if there is an embedded compact orientable surface $S$ in $X \times I$ such that $V \subseteq \partial S$ and there is a partial homotopy $H: X \times I \times\{0\} \cup S \times I \rightarrow X$ satisfying: $H(x, 0)=F(x)$ for all $x \in X \times I, H(x, 1)=x$ for all $x \in S$, and $H(x, t)=x$ for all $x \in V$ and all $t$.

Now we recall a variation of Theorem 9.8 from [DG].

Theorem 9.8 [DG]. Let $F: X \times I \rightarrow X$ be transverse to $P$ with no fixed points in $\partial(X \times I)$ and transverse fixed point set. Let $V=$ $\cup C_{j}$ be a union of circles of fixed points such that $V^{\prime}$ lies in a path component of $E$. If the geometric 1-cycle in $E$ defined by $V^{\prime}$ with the natural orientations on the $C_{j}$ 's is $\mathbb{Z}$-homologous to zero, and if the connected surface $S$ spanning $V$ can be chosen with trivial associated $\mathbb{Z}_{2}$ obstruction, then there is a neighborhood, $N$, of $V$ in $\operatorname{int}(X \times I)$ containing no other fixed points of $F$, and a homotopy from $F$ to $H \operatorname{rel} X \times I \backslash N$, such that $\operatorname{Fix}(H)=\operatorname{Fix}(F) \backslash V$.

Theorem 10.1 in [DG] shows that the vanishing of the obstructions is not only sufficient, but also necessary. 
THeOREM 10.1 [DG]. With the notation as in Theorem 9.8, let there exist a compact neighborhood $N$ of $V$ in $\operatorname{int}(X \times I)$ containing no other fixed points of $F$ and, a homotopy between $F$ and $G, \operatorname{rel} X \times$ $I \backslash N$, such that $\operatorname{Fix}(G)=\operatorname{Fix}(F) \backslash V$. Then there is a connected surface $S$, spanning $V$ with a partial homotopy as above, whose associated $\mathbb{Z}_{2}$-obstruction vanishes.

Now we improve both of these theorems by the following:

Theorem 4.1. Let $F: X \times I \rightarrow X$ be tranverse to $P$ with no fixed points in $\partial(X \times I)$ and transverse fixed point set as in [DG]. Let $V=$ $\cup C_{j}$ be a union of isolated circles of fixed points, such that $V^{\prime}$ lies in a path component of $E$. Then, there is a neighborhood, $N$, of $V$ in $\operatorname{int}(X \times I)$ containing no other fixed points of $F$, and a homotopy from $F$ to $H$ rel $X \times I \backslash N$, such that $\operatorname{Fix}(H)=\operatorname{Fix}(F) \backslash V$, if and only if: the geometric 1-cycle in $E$ defined by $V^{\prime}$ with the natural orientations on the $C_{j}$ 's is $\mathbb{Z}$-homologous to zero; and $\sum i_{2}\left(F, C_{j}\right)=0$.

Proof. The proof is similar to the proof in [DG]. Let the geometric 1-cycle determined by $V^{\prime}$ be $\mathbb{Z}$-homologous to zero and let $\sum i_{2}\left(F, C_{j}\right)=0$. The fact that the geometric 1-cycle determined by $V^{\prime}$ is zero implies the existence of an embedded, oriented, compact, connected surface $S \subseteq \operatorname{int}(X \times I)$ spanning $V$ and inducing the natural orientation on the circles in $V$, together with a partial homotopy $K:(X \times I \times\{0\}) \cup(S \times I) \rightarrow X$, with $K(x, 0)=F(x)$ for all $x \in X \times I, K(x, 1)=x$ for all $x \in S$, and $K(x, t)=x$ for all $x \in V$ and all $t$ ([DG]). Then by the cutting argument given in [DG], and by Propositions 3.9 and $3.10, S$ can be reduced to a disk $B$. This cutting argument gives a homotopy from $F$ to a map $G \operatorname{rel} X \times I \backslash N^{\prime}$ for a neighborhood $N^{\prime}$ of $V$, such that: $\operatorname{Fix}(G)=(\operatorname{Fix}(F) \backslash V) \cup \partial B$; $i_{1}(G, \partial B)=1$; and $i_{2}(G, \partial B)=\sum i_{2}\left(F, C_{i}\right)=0$. Further, by Proposiy tion 3.4, $G$ is homotopic to a map $H \operatorname{rel}(X \times I) \backslash N^{\prime \prime}$ for a neighborhood $N^{\prime \prime}$ of $B$, such that $\operatorname{Fix}(H)=\operatorname{Fix}(G) \backslash \partial B=\operatorname{Fix}(F) \backslash V$.

Conversely, suppose there exist a neighborhood $N$ of $V$ and a homotopy from $F$ to $H \operatorname{rel} X \times I \backslash N$, such that $\operatorname{Fix}(H)=\operatorname{Fix}(F) \backslash V$. Then as it is shown in [DG], the 1-cycle in $E$ determined by $V^{\prime}$ is $\mathbb{Z}$ homologous to zero, and there exists a surface $S$ as above, whose associated $\mathbb{Z}_{2}$-obstruction is zero. But the associated $\mathbb{Z}_{2}$-obstruction for $S$ is obtained after the cutting argument, which by Propositions 3.9 and 3.10 , implies that the $\mathbb{Z}_{2}$-obstruction for $S$ is equal to $\sum i_{2}\left(F, C_{j}\right)$, and hence, $\sum i_{2}\left(F, C_{j}\right)=0$. 
In the above theorems we used repeatedly a family of isolated transverse circles of fixed points $V$ and its corresponding family $V^{\prime}$ in the space of paths $E$. Next we generalize this notion slightly. Let $V$ be a family of isolated circles of fixed points (not necessarily transverse) and isolated fixed points (which cannot be transverse). For such a set $V$, let $V^{\prime}$ be the subset of $E$ defined as above using constant paths. Then $V^{\prime}$ is a family of circles and points in $E$. A set $V$ is in a single fixed point class if and only if $V^{\prime}$ is in a single path component of $E$. For an isolated oriented circle of fixed points $C$, let $\left\{C^{\prime}\right\}$ be the element from $H_{1}(E)$ determined by the geometric 1-cycle $C^{\prime}$. For such sets $V$ we define two indices.

DEFINITION 4.1. Let $V$ be a family of isolated circles of fixed points $C_{1}, \ldots, C_{k}$ (not necessarily tranverse) and isolated fixed points $A_{1}, \ldots, A_{r}$ in a single fixed point class. Let $C_{j}$ be oriented by the natural orientation. We define the index 1 of $V$, denoted by ind $_{1}(F, V)$, to be the element $\sum i_{1}\left(F, C_{j}\right) \cdot\left\{C_{j}^{\prime}\right\}$ in $H_{1}(E)$. We define the index 2 of $V$, denoted by $\operatorname{ind}_{2}(F, V)$, to be the element $\sum i_{2}\left(F, C_{j}\right)+\sum i_{2}\left(F, A_{i}\right)$ in $\mathbb{Z}_{2}$.

The improvement in Theorem 4.1 is the fact that the $\mathbb{Z}_{2}$ obstruction does not depend on the surface. But there is another improvement. In [DG] the cutting argument was not producing new circles of fixed points, and here the cutting argument is producing new circles of fixed points. The generalization of this is the following theorem.

THEOREM 4.2. Let $V$ be a family of isolated circles of fixed points and isolated fixed points of $F$ in a single fixed point class. Then there is a neighborhood $N$ of $V$ missing other fixed points and a homotopy from $F$ to $G \operatorname{rel} X \times I \backslash N$, such that: $\operatorname{Fix}(G)=(\operatorname{Fix}(F) \backslash V) \cup C$, where $C$ is an isolated circle of fixed points of $G ; \operatorname{ind}_{1}(G, C)=$ 0 if and only if $\operatorname{ind}_{1}(F, V)=0$; and $\operatorname{ind}_{2}(G, C)=\operatorname{ind}_{2}(F, V)$. Moreover, the circle $C$ can be chosen to be transverse, which implies that $i_{1}(G, C)=1$.

Proof. The proof consists of the following steps.

Step 1. Conclusion (4) of Proposition 3.6 implies that $F$ is homotopic to a map $F_{1} \operatorname{rel} X \times I \backslash N_{1}$ where $N_{1}$ is a small compact neighborhood of the circles of fixed points $C_{j}$ with $i_{1}\left(F, C_{j}\right)=0$, such that: these circles are replaced by fixed points; the new family $V_{1}$ is in a single fixed point class of $F_{1}$; ind ${ }_{1}\left(F_{1} ; V_{1}\right)=0$ if and only if $\operatorname{ind}_{1}(F, V)=0$; and $\operatorname{ind}_{2}\left(F_{1}, V_{1}\right)=\operatorname{ind}_{2}(F, V)$. 
Step 2. Proposition 3.7 implies that $F_{1}$ is homotopic to a map $F_{2}$ rel $X \times I \backslash N_{2}$, where $N_{2}$ is a small compact neighborhood of the fixed points $A_{j}$ in $V_{1}$, such that: the fixed points $A_{j}$ are replaced by circles of fixed points $K_{j}$; the new family $V_{2}$ is in a single fixed point class of $F_{2}$; each geometric 1-cycle $K_{j}^{\prime}$ in $E$ determined by $K_{j}$ is nullhomotopic, and moreover, $\mathbb{Z}$-homologous to zero; $K_{j}$ are transverse and $i_{1}\left(F_{2}, K_{j}\right)=1$; and $i_{2}\left(F_{2}, K_{j}\right)=i_{2}\left(F_{1}, A_{j}\right)$. This implies that $\operatorname{ind}_{1}\left(F_{2}, V_{2}\right)=0$ if and only if $\operatorname{ind}_{1}(F, V)=0$, and $\operatorname{ind}_{2}\left(F_{2}, V_{2}\right)=\operatorname{ind}_{2}(F, V)$.

Step 3. Proposition 3.6 implies that $F_{2}$ is homotopic to $F_{3} \operatorname{rel} X \times$ $I \backslash N_{3}$, where $N_{3}$ is a small compact neighborhood of $V_{2}$, such that: each circle $C_{j}$ from $V_{2}$ is replaced by $i_{1}\left(F_{2}, C_{j}\right)$ circles; the family $V_{3}$ is in a single fixed point class; for each circle $C_{j}$ in $V_{3}, C_{j}$ is transverse and $i_{1}\left(F_{3}, C_{j}\right)=1$; $\operatorname{ind}_{1}\left(F_{3}, V_{3}\right)=0$ if and only if $\operatorname{ind}_{1}(F, V)=0 ;$ and $\operatorname{ind}_{2}\left(F_{3}, V_{3}\right)=\operatorname{ind}_{2}(F, V)$.

Step 4. We reduce the number of circles of fixed points in $V_{3}$ by induction. Let $C_{1}, C_{2}$ be two circles in $V_{3}$. Since they are in a single fixed point class there is an arc $\alpha$ from $C_{1}$ to $C_{2}$ and a homotopy from $F_{3} \circ \alpha$ to $P \circ \alpha$, i.e. a partial homotopy $H^{\prime}: X \times I \times\{0\} \cup \alpha(I) \times I \rightarrow$ $X$ from $F_{3}$ to $P$. The partial homotopy can be extended to a partial homotopy $H: X \times I \times\{0\} \cup \varphi(K G) \times I \rightarrow X$ where $\varphi: K G \rightarrow X \times I$ is an embedding such that: $\varphi(K G)$ is contained in a small regular neighborhood $N_{4}$ of $C_{1} \cup C_{2} \cup \alpha(I) ; \varphi\left(K_{2}\right)=C_{1} ; \varphi\left(K_{3}\right)=C_{2}$; and the orientation on $C_{j}$ induced from the orientation on $K G$ via $\varphi$ agrees with the natural orientation. Then Proposition 3.9 implies that $F_{3}$ is homotopic to a map $F_{4} \operatorname{rel} X \times I \backslash N_{4}$ such that: the family $V_{4}=$ $\left(V_{3} \backslash\left\{C_{1}, C_{2}\right\}\right) \cup\left\{\varphi\left(K_{1}\right)\right\}$ is in a single fixed point class; for each circle $C_{j}$ in $V_{4}, C_{j}$ is transverse and $i_{1}\left(F_{4}, C_{j}\right)=1 ; \operatorname{ind}_{1}\left(F_{4}, V_{4}\right)=0$ if and only if $\operatorname{ind}_{1}(F, V)=0$; and $\operatorname{ind}_{2}\left(F_{4}, V_{4}\right)=\operatorname{ind}_{2}(F, V)$. The same process can be applied to $F_{4}$, so that at the end we will obtain a map $G$ which satisfies all the conclusions of the Theorem.

We note that Step 4 can be applied directly to $F$ and $V$, obtaining all the conclusions of the theorem except the moreover part, if $V$ consists only of isolated circles of fixed points and if there is a number $s \geq 0$ such that, for each circle $C_{j}$ in $V, i_{1}\left(F, C_{j}\right)=s$.

Next we give another improvement of Theorem 4.1.

THEOREM 4.3. Let $X \subseteq \mathbb{R}^{m}$ be an m-dimensional compact connected orientable manifold, $m \geq 4$, let $F: X \times I \rightarrow X$, and let 
$V \subseteq \operatorname{int}(X \times I)$ be a family of isolated circles of fixed points and isolated fixed points of $F$ in a single fixed point class. Then, there exists a compact neighborhood $N$ of $V$ and a homotopy from $F$ to $G \operatorname{rel} X \times I \backslash N$ such that $\operatorname{Fix}(G)=\operatorname{Fix}(F) \backslash V$ if and only if $\operatorname{ind}_{1}(F, V)=0$ in $H_{1}(E)$, and $\operatorname{ind}_{2}(F, V)=0$ in $\mathbb{Z}_{2}$.

Proof. The proof follows from Theorems 4.2 and 4.1.

Definition 4.2. Let $F$ have only isolated circles of fixed points and isolated fixed points. A fixed point class $V$ is said to be inessential if $\operatorname{ind}_{1}(F, V)=0$ and $\operatorname{ind}_{2}(F, V)=0$, and essential otherwise. We denote by $N(F)$ the number of essential fixed point classes.

Finally, we state the following theorem about the number and types of fixed point classes whose proof follows from the above results. Let $F, X$ be as above, and $m \geq 4$.

TheOREM 4.4. (1) $F$ is homotopic to a map $G_{1}$ such that $G_{1}$ has exactly $N(F)$ fixed point classes.

(2) $F$ is homotopic to a map $G_{2}$ such that $G_{2}$ has exactly $N(F)$ isolated circles of fixed points.

(3) $F$ is homotopic to a map $G_{3}$ such that $G_{3}$ has exactly $N(F)$ isolated transverse circles of fixed points.

(4) If $F$ is homotopic to a map $H$ such that $H$ has only isolated circles of fixed points and isolated fixed points, then the number of fixed point classes of $H$ is bigger than or equal to $N(F)$.

\section{REFERENCES}

[BJ] B. Jiang, Fixed point classes from a differential viewpoint, in Fixed Point Theory, Springer Lecture Notes in Mathematics, Vol. 886, (1981), 163-170.

[B] Lectures on Nielsen fixed point theory, Contemp. Math., vol. 14, Amer. Math. Soc., Providence, RI, 1983.

[DG] D. Dimovski and R. Geoghegan, One-parameter fixed point theory, Forum Math., 2 (1990), 125-154.

[Ha] V. L. Hansen, The homotopy problem for the components in the space of maps on the n-sphere, Quart. J. Math., Oxford (3), 25 (1974), 313-321.

[HQ] A. Hatcher and F. Quinn, Bordism invariants of intersections of submanifolds, Trans. Amer. Math. Soc., 200 (1974), 327-344.

[H] P. Hilton, Homotopy Theory and Duality, Gordon and Breach, New York, 1965.

[J] J. Jezierski, One codimensional Wecken type theorems, to appear.

[RS] C. Rourke and B. Sanderson, Piecewise Linear Topology, Springer-Verlag, New York, Heidelberg, Berlin 1972. 
[S] H. Schirmer, Fixed point sets of homotopies, Pacific J. Math., 108 (1983), 191-202.

[W] G. Whitehead, Elements of Homotopy Theory, Springer-Verlag, New York, Heidelberg, Berlin, 1978.

Received March 12, 1992 and in revised form, November 20, 1992. Partially supported by a grant from the Research Council of Macedonia.

UNIVERSITY OF SKOPJE

PF 162, 91000 SKOPJE

MACEDONIA 



\section{CONTENTS}

K. Auinger, Free products of combinatorial strict inverse semigroups . . . . . . . . 201

T. Dana-Picard, Generic 8-dimensional algebras with mixed basis-graph . . . . . . 229

D. Dimovski, One-parameter fixed point indices . . . . . . . . . . . . . . . . . 263

K. S. Druschel, Oriented orbifold cobordism . . . . . . . . . . . . . . . . . . . 299

A. D. Helfer, Conjugate points on spacelike geodesics or pseudo-self-adjoint MorseSturm-Liouville systems . . . . . . . . . . . . . . . . . . . . . 321

J. G. Heywood and O. D. Walsh, A counter-example concerning the pressure in the Navier-Stokes equations, as $t \rightarrow 0^{+} \ldots \ldots \ldots \ldots \ldots \ldots \ldots \ldots \ldots, \ldots \ldots \ldots$

G. Masbaum and P. Vogel, 3-valent graphs and the Kauffman bracket . . . . . . 361

R. Stong, The Jones polynomial of parallels and applications to crossing number 383

Vol. 164, No. 2 


\section{PACIFIC JOURNAL OF MATHEMATICS}

Volume $164 \quad$ No. $2 \quad$ June 1994

Free products of combinatorial strict inverse semigroups

201

KARL AUINGER

Generic 8-dimensional algebras with mixed basis-graph

229

THIERRY DANA-PICARD

One-parameter fixed point indices

263

DONCO DIMOVSKI

Oriented orbifold cobordism

299

KIMBERLY SUE DRUSCHEL

Conjugate points on spacelike geodesics or pseudo-self-adjoint

Morse-Sturm-Liouville systems

ADAM D. HELFER

A counterexample concerning the pressure in the Navier-Stokes

351 equations, as $t \rightarrow 0^{+}$

JOHN GROVES HeYwOOD and OWEN WALSH

3-valent graphs and the Kauffman bracket

Gregor Masbaum and PierRe Vogel

The Jones polynomial of parallels and applications to crossing number 383

RICHARD ANDREW STONG 